\title{
Multiple stellar populations in the globular cluster NGC $1851^{\star, \star \star}$
}

\author{
E. Carretta ${ }^{1}$, S. Lucatello ${ }^{2}$, R. G. Gratton ${ }^{2}$, A. Bragaglia ${ }^{1}$, and V. D'Orazi ${ }^{2}$ \\ ${ }^{1}$ INAF - Osservatorio Astronomico di Bologna, via Ranzani 1, 40127 Bologna, Italy \\ e-mail: eugenio.carretta@oabo.inaf.it \\ 2 INAF - Osservatorio Astronomico di Padova, Vicolo dell'Osservatorio 5, 35122 Padova, Italy \\ Received 17 May 2011 / Accepted 16 June 2011
}

\begin{abstract}
We present a detailed chemical tagging of individual stellar populations in the Galactic globular cluster (GC) NGC 1851. Abundances are derived from FLAMES spectra for the largest sample of giants (124) and the most extensive number of elements ever analysed in this peculiar GC. The chemistry is characterised using homogeneous abundances of proton-capture $(\mathrm{O}, \mathrm{Na}, \mathrm{Mg}, \mathrm{Al}, \mathrm{Si}), \alpha$-capture (Ca, Ti), Fe-peak (Sc, V, Mn, Co, Ni, Cu), and neutron-capture elements (Y, Zr, Ba, La, Ce, Nd, Eu, Dy). We confirm the presence of an $[\mathrm{Fe} / \mathrm{H}]$ spread larger than the observational errors in this cluster, but too small to clearly separate different sub-populations. We instead propose a classification scheme using a combination of $\mathrm{Fe}$ and $\mathrm{Ba}$ (which is much more abundant in the more metal-rich group) by means of a cluster analysis. With this approach, we separated stars into two components of a metal-rich (MR) and a metal-poor (MP) population. Each component displays a Na-O anticorrelation, which is a signature of a genuine GC, but has different ratios of primordial (FG) to polluted (SG) stars. Moreover, clear (anti)correlations of $\mathrm{Mg}$ and $\mathrm{Si}$ with $\mathrm{Na}$ and $\mathrm{O}$ are found for each component. The level of $[\alpha / \mathrm{H}]$ tracks iron and is higher in the MR population, which might therefore have received an additional contribution from core-collapse supernovae. When considering all $s$-process elements, the MR population shows a larger enrichment than the MP one. This is probably due to the contribution of intermediate-low mass stars, because we find that the level of heavy s-process elements is higher than that of light $s$-process nuclei in the MR stars; however, a large contribution from low mass stars is unlikely, because it would likely cancel the O-Na anticorrelation. Finally, we confirm the presence of correlations between the amount of proton-capture elements and the level of $s$-process elements previously found by other investigations, at least for the MR population. This finding apparently requires a quite long delay for the second generation of the MR component. Scenarios for the formation of NGC 1851 appear complex, and are not yet well understood. A merger of two distinct GCs in a parent dwarf galaxy, each cluster with a different Ba level and an age difference of $\sim 1 \mathrm{Gyr}$, might explain (i) the double subgiant branch; (ii) a possible difference in C content between the two original GCs; and (iii) the Strömgren photometry of this peculiar cluster. However, the correlation existing between $p$-capture and $n$-capture elements within the MR population requires the additional assumption of a long delay for its second generation. More observations are required to fully understand the formation of this GC.
\end{abstract}

Key words. stars: abundances - stars: atmospheres - globular clusters: general - globular clusters: individual: NGC 1851 stars: Population II

\section{Introduction}

Spectroscopy (for several decades) and more recently photometry (in particular in the near ultraviolet window) have shown that globular clusters (GCs) are much more complex than previously imagined. Since the publishing of the pioneering work by Cohen (1978) and Peterson (1980), more than 30 years of abundance analysis of GC stars have shown that they host multiple populations, with slightly different ages but a wide range of different chemical composition (see Kraft 1994; and Gratton et al. 2004, for reviews and references). The most outstanding signature of a GC is the observed $\mathrm{Na}-\mathrm{O}$ anticorrelation, which has been detected so far in every $G C$ investigated with high resolution, high quality spectra ${ }^{1}$. This feature is so widespread that it is

\footnotetext{
* Based on observations collected at ESO telescopes under programme 083.D-0208.

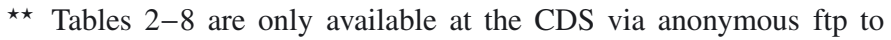
cdsarc.u-strasbg.fr $(130.79 .128 .5)$ or via

http://cdsarc.u-strasbg.fr/viz-bin/qcat?J/A+A/533/A69

${ }^{1}$ In GCs in which the anti-correlation was not found, Pal12 and Ter 7 (Sbordone et al. 2007; Cohen 2004, respectively) only a few stars were studied, hence small number statistics prevents the drawing of any conclusion.
}

interpreted as the birthmark of genuine, bona fide GCs (Carretta et al. 2010a), likely related to their very own formation mechanism(s) (Carretta 2006). Although the exact chain of events leading to the GCs we observe is still in a "tentative scenario" stage, some key points are currently clear.

The enhancement in $\mathrm{Na}$ (and $\mathrm{N}, \mathrm{Al}$ ) and the depletion in $\mathrm{O}$ (and $\mathrm{C}, \mathrm{Mg}$ ) are also observed in unevolved stars (e.g. Gratton et al. 2001), where it provides evidence of a previous generation of more massive stars that ended their relatively short lifetimes with the injection of nuclear-processed matter in the intra-cluster medium. From the mix of pristine material with these enriched ejecta (see e.g. Prantzos \& Charbonnel 2006), a second generation of stars is born. The typical shape of the Na-O anticorrelation is then probably due to some dilution with pre-existing gas of the ashes from proton-capture reactions in H-burning at quite high temperatures (Denisenkov \& Denisenkova 1989; Langer et al. 1993). The degree of the processing is modulated by some combination of global cluster parameters, the critical ones being total mass and metallicity (Carretta et al. 2010a). This is basically the scenario based on the operation of early polluters in the first phases of cluster life, although the exact nature of these polluting stars is still debated (massive, rapidly rotating stars, Decressin et al. 2007; versus intermediate-mass 
asymptotic branch AGB stars, Ventura et al. 2001, are currently the main candidates).

However, our ongoing extensive FLAMES survey of the $\mathrm{Na}-\mathrm{O}$ anticorrelation in more than 20 GCs (see Carretta et al. 2006, 2009a-c, 2010b,c; Gratton 2010a, for strategy and the updated status of the survey) has revealed that superimposed on this common pattern, there is in some cases one (or more) further degrees of complexity. Even neglecting the likely remnants of dispersed/dispersing dwarf galaxies (such as M 54 and possibly $\omega$ Cen, Carretta et al. 2010b), the globular cluster NGC 1851 stands out.

NGC 1851 has been known for a long time to show a bimodal colour distribution of stars in the core He-burning phase (e.g. Walker 1992): its horizontal branch (HB) is discontinuous, consisting of a well populated red HB (RHB), a few RR Lyrae stars, and an extended blue HB (BHB; Fig. 1). Despite being somewhat less massive than clusters displaying distinct multiple main-sequences (such as NGC 2808 and $\omega$ Cen, D'Antona et al. 2005; and Anderson 1998, respectively), NGC 1851 was found to contain two subgiant branches (SGBs; Milone et al. 2008) with a magnitude difference corresponding to about $1 \mathrm{Gyr}$, if interpreted as an age difference. The same division may however be explained by a spread in the total CNO abundances (Cassisi et al. 2008); such a spread was claimed by Yong et al. (2009) to exist in NGC 1851 from the analysis of four red giant branch (RGB) stars, while a constant sum of $\mathrm{C}+\mathrm{N}+\mathrm{O}$ abundances was derived for a sample of 15 RGB stars by Villanova et al. (2010, hereinafter V10).

The same level of luminosity shared by the RHB and the BHB seems to exclude there being a large range of He among stellar populations in NGC 1851 (Cassisi et al. 2008). On the other hand, the lack of division in the MS implies that the two sequences have a similar $[\mathrm{Fe} / \mathrm{H}]^{2}$. However, this does not exclude the presence of a small metallicity spread. Both the population ratios and the central concentration led some studies to connect the RHB to the younger SGB and the extended BHB to the older SGB, in the framework of an age difference. However, the issue of a different radial distribution was still debated until recently (Zoccali et al. 2009; Milone et al. 2009). Our observations of a small metallicity spread and of a different distribution of the most metal-poor and metal-rich component on the RGB (Carretta et al. 2010c) finally provided conclusive evidence that two stellar populations, differently concentrated within the cluster, do co-exist in NGC 1851.

The finding of a small but we believe real metallicity dispersion in NGC 1851 was immediately compared with the split of the RGB uncovered by Lee et al. (2009a) using narrow-band Ca II photometry and by Han et al. (2009) with UVI Johnson broadband photometry. In turn, this might be interpreted in the context of the scenario devised by Lee et al. (2009b), who claimed GCs with multiple populations had experienced an enrichment by supernovae (SNe). While Carretta et al. (2010d) confuted that $\mathrm{SNe}$ provided this contribution to all GCs, the reality of different levels of $\mathrm{Ca}$ and other $\alpha$-elements from corecollapse SNe could not be excluded for this particular cluster.

To complicate the sitution yet further, Yong \& Grundahl (2008) found variations in $\mathrm{Zr}$ and $\mathrm{La}$ that are correlated with those of $\mathrm{Al}$, suggesting some contribution from thermally pulsing AGB stars. This introduces another problem because light $s$-process elements and $\mathrm{Al}$ are thought to be produced by stars

\footnotetext{
${ }^{2}$ We adopt the usual spectroscopic notation, i.e. for any given species $\mathrm{X},[\mathrm{X}]=\log \epsilon(X)_{\mathrm{star}}-\log \epsilon(X)_{\odot}$ and $\log \epsilon(X)=\log \left(N_{\mathrm{X}} / N_{\mathrm{H}}\right)+$ 12.0 for absolute number density abundances.
}

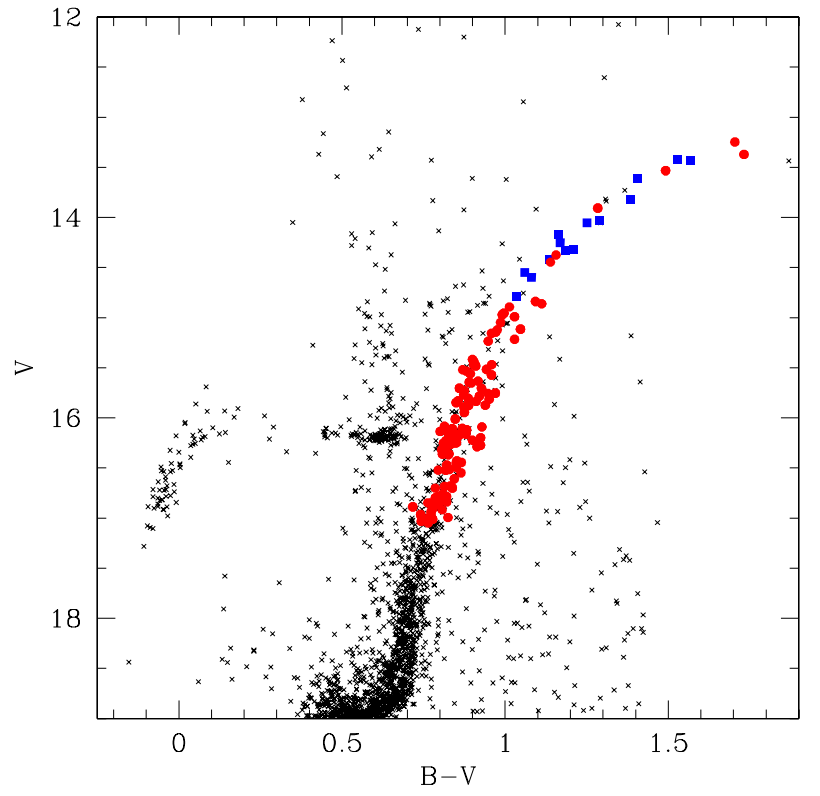

Fig. 1. The $V, B-V$ CMD of NGC 1851 with stars selected for the present study indicated by large red filled circles (GIRAFFE targets) and large blue squares (UVES targets).

of different mass ranges. When different mass ranges are involved, some time delay and/or prolonged star formation must also be taken into account, increasing the level of complexity in any possible modelling of the earliest phase of cluster formation and evolution.

To deal with all the old and new pieces of the "puzzle NGC 1851", we tentatively proposed in Carretta et al. (2010c) a scenario that may account for all the observations there considered. According to this scenario, the current cluster is the result of a merger between two distinct clusters born in a much larger system, such as a dwarf galaxy now no longer visible. A subset of elements was used to show that this hypothesis, already put forward by some authors (van den Bergh 1996; Catelan 1997), is able to accommodate most of the observations.

Here we present our entire large dataset consisting of abundances for many species derived in more than 120 red giants in NGC 1851. This dataset will be discussed within this framework. The paper is organised as follow. Our data and analysis techniques are presented in Sects. 2 and 3, with particular emphasis on neutron-capture elements, whose abundances play an important role in this GC but were not considered in detail in Carretta et al. (2010c). The metallicity spread and the operative definition of the stellar components in NGC 1851 are discussed in Sect. 4. The complex nucleosynthesis observed in this GC is examined in detail in Sect. 5, while an extensive comparison of results from spectroscopy and photometry is presented in Sect. 6 . Finally, these data are discussed in Sect. 7, where some tentative conclusion will be drawn. The Appendix gives detail about the error analysis.

\section{Sample selection and observations}

The sample of RGB stars to be observed in NGC 1851 was selected with the same criteria (small distance from RGB ridge line, absence of close companions) adopted in previous works 
Table 1. Log of FLAMES observations for NGC 1851.

\begin{tabular}{lccccc}
\hline \hline Date & UT & $\begin{array}{c}\text { Exp. } \\
(\mathrm{s})\end{array}$ & Grating & Seeing & Airmass \\
& & & & \\
\hline Apr. 09, 2010 & $23: 53: 06.585$ & 2880 & HR11 & 0.82 & 1.355 \\
Apr. 11, 2010 & $00: 21: 43.120$ & 2880 & HR11 & 0.67 & 1.514 \\
Aug. 05, 2010 & $09: 13: 30.637$ & 2880 & HR11 & 1.02 & 1.533 \\
Aug. 26, 2010 & $07: 57: 12.771$ & 2880 & HR13 & 1.21 & 1.497 \\
Aug. 26, 2010 & $08: 47: 34.969$ & 2880 & HR13 & 1.11 & 1.278 \\
Sep. 01, 2010 & $08: 00: 05.991$ & 2880 & HR13 & 1.78 & 1.368 \\
Sep. 02, 2010 & $07: 41: 33.444$ & 2880 & HR13 & 1.35 & 1.435 \\
\hline
\end{tabular}

of our survey. FLAMES observations (the same also used in Carretta et al. 2010c) consisted of three pointings with the HR11 high resolution grating $(R=24200)$ covering the Na I 5682-88 $\AA$ doublet and four pointings with the grating HR13 $(R=22500)$ including the [O I] forbidden lines at 6300-63 A. The median signal-to-noise (S/N) ratio is about 75 . The observation log is listed in Table 1 and the selected stars are shown on a $V, B-V$ colour-magnitude diagram (CMD) in Fig. 1.

$V$ and $B$ band photometry (kindly provided by Y. Momany) derived from ESO Imaging Survey images was complemented with near infrared photometry, to obtain atmospheric parameters as described below. $K$ band magnitudes were obtained from the Point Source Catalogue of 2MASS (Skrutskie et al. 2006) and properly transformed to the TCS photometric system, to be used to derive input atmospheric parameters, following the Alonso et al. (1999) calibration (see below).

Initially, 124 member stars (according to the radial velocity $\mathrm{RV}$, see below) were targeted with GIRAFFE fibres and 14 stars with the fibre-fed UVES spectrograph (Red Arm, with spectral range from 4800 to $6800 \AA$ and $R=45000$ ); 10 stars were observed with both instruments.

We used the one-dimensional, wavelength-calibrated spectra as reduced by the dedicated ESO Giraffe pipeline to derive RVs using the IRAF ${ }^{3}$ task FXCOR, with appropriate templates, while the RVs of the stars observed with UVES were derived with the IRAF task RVIDLINES.

Three stars with GIRAFFE spectra and one with UVES spectra were successively dropped from the sample, because of unreliable photometry and/or spectra. Hence, our final sample for NGC 1851 consists of 124 RGB stars, the largest data set ever observed with high resolution spectroscopy in this cluster. Coordinates, magnitudes, and heliocentric RVs are shown in Table 2 (the full table is only available in electronic form at CDS).

\section{Atmospheric parameters and abundance analysis}

We derived the atmospheric parameters and abundances using the same technique adopted for the other GCs targeted by our FLAMES survey. In particular, since we believe we have detected a small metallicity spread in NGC 1851, the reference paper where the tools are described in detail is the analysis of M 54 (Carretta et al. 2010e), although in that case the spread in $[\mathrm{Fe} / \mathrm{H}]$

\footnotetext{
3 IRAF is distributed by the National Optical Astronomical Observatory, which are operated by the Association of Universities for Research in Astronomy, under contract with the National Science Foundation.
}

is much larger. Therefore, only a few points of the analysis will be summarised here.

As usual, we derived first estimates of $T_{\text {eff }}$ (from $V-$ $K$ colours) and bolometric corrections using the calibrations of Alonso et al. (1999, 2001). Subsequently, we refined the $T_{\text {eff }}$ estimates for the stars using a relation between $T_{\text {eff }}$ and $K$ magnitudes ${ }^{4}$. Gravities were obtained from stellar masses and radii, these last two parameters being derived from luminosities and temperatures. Within this procedure, the reddening and the distance modulus for NGC 1851 were taken from the Harris (1996) catalogue (2011 update), and we adopted masses of $0.85 M_{\odot}$ for all stars ${ }^{5}$ and $M_{\mathrm{bol}, \odot}=4.75$ as the bolometric magnitude for the Sun, as in our previous studies.

The abundance analysis is mainly based on equivalent widths $(E W \mathrm{~s})$. Those measured in GIRAFFE spectra were corrected to the system given by UVES spectra. Values of the microturbulent velocities $v_{\mathrm{t}}$ were obtained by eliminating trends between $\mathrm{Fe}_{\mathrm{I}}$ abundances and expected line strength (Magain 1984). Finally, we interpolated models with the appropriate atmospheric parameters whose abundances matched those derived from Fe I lines within the Kurucz (1993) grid of model atmospheres (with the option for overshooting on). The adopted atmospheric parameters and iron abundances are listed in Table 3.

The procedure for error estimates is the same as in Carretta et al. (2010e) and results are given in the Appendix for UVES and GIRAFFE observations in Tables A.1 and A.2, respectively.

As in our previous papers, oxygen abundances are obtained from the forbidden [O I] lines at 6300.3 and $6363.8 \AA$, after cleaning the former from telluric lines as described in Carretta et al. (2007c). The prescription of Gratton et al. (1999) was adopted to correct the derived $\mathrm{Na}$ abundances for non-LTE effects (from the 5682-88 and 6154-60 ̊ doublets). The abundances of $\mathrm{Al}$ (from the 6696-99 $\AA$ doublet) and $\mathrm{Mg}$ are derived as in Carretta et al. (2009b). The abundances of these protoncapture elements for individual stars are listed in Table 4.

The observed $\alpha$-elements in the present study are $\mathrm{Mg}$ (reported among proton-capture elements), $\mathrm{Si}, \mathrm{Ca}$, and $\mathrm{Ti}$. The abundances of these elements were derived for stars with either UVES or GIRAFFE spectra (see Table 5). For the first ones, we measured Ti abundances from both neutral and singly ionised species, owing to the large spectral coverage of UVES spectra ${ }^{6}$.

To investigate the Fe-peak elements, we measured lines of Sc II, V I, Cr I, Co I, and Ni I for stars with GIRAFFE and UVES spectra and in addition of $\mathrm{Mn} \mathrm{I}$ and $\mathrm{Cu}$ I for stars with UVES spectra. Whenever relevant (e.g. Sc, V, Mn, Co), we applied corrections for the hyperfine structure (see Gratton et al. 2003, for references). For $\mathrm{Cu}$ I, we used the transition at $5105 \AA$, which we synthesised using a line list kindly provided by J. Sobeck in advance of publication. The line list takes into account both isotopic structure and hyperfine splitting. We adopted the solar isotopic ratio of $69 \%{ }^{63} \mathrm{Cu}$ and $31 \%{ }^{65} \mathrm{Cu}$. Abundances of Fe-peak elements for individual stars are reported in Table 6.

We derived the concentration of several neutron-capture elements, mostly from UVES spectra. We measured the abundances of Y II, Zr II, Ba II, La II, Ce II, Nd II, Eu II, and Dy II using a

\footnotetext{
${ }^{4}$ For a few stars with no $K$ in 2MASS, we used $K$ values obtained by interpolating a quadratic relation as a function of $V$ magnitudes.

5 Values of surface gravity, derived from the positions of stars in the CMD, are not very sensitive to the exact value of the adopted mass.

6 The very good agreement obtained for Ti I and $\mathrm{Ti}$ in in Table 9 supports our adopted scale of atmospheric parameters, since the ionization balance is very sensitive to any detail, and error, in the abundance analysis.
} 

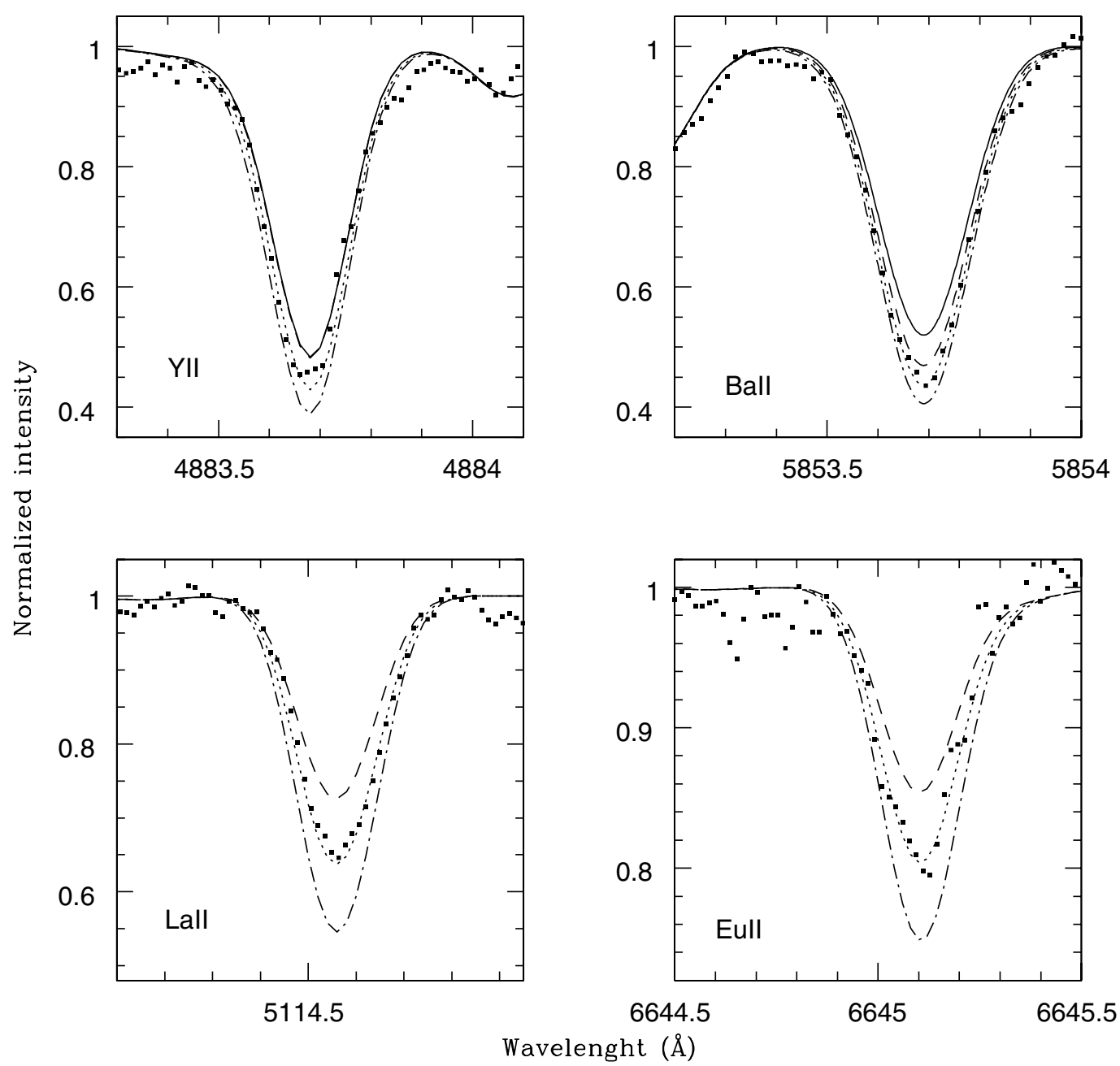

Fig. 2. Examples of syntheses for lines due to four $n$-capture species in star 14080 . Upper left side: syntheses for the $\mathrm{Y}_{\text {II }}$ line at $4883 \AA$, the abundances plotted are $[\mathrm{Y} / \mathrm{Fe}]_{\mathrm{II}}=0.1,0.3$, and 0.5 dex. Upper right side: as upper left for the $\mathrm{Ba}$ II line at $5853.5 \AA$ for $[\mathrm{Ba} / \mathrm{Fe}]_{\mathrm{II}}=0.30,0.50$, and 0.70 dex. Lower right side: as upper left for the Eu line at $6645.1 \AA$ for $[\mathrm{Eu} / \mathrm{Fe}]_{\mathrm{II}}=0.4,0.6$, and 0.8 ; lower left side: as for upper left for the La II line $5114.5 \AA$ for $[\mathrm{La} / \mathrm{Fe}] \mathrm{II}=0.2,0.4$, and $0.6 \mathrm{dex}$. For Y and Ba, the solid lines indicate the values reported in V10 for this star.

combination of spectral synthesis and $E W$ measurements. A few details follow for each element:

Y II: we used four transitions (at 4883, 5085, 5200, $5206 \AA$ ). They were synthesised using the same atomic parameters as listed in Sneden et al. (2003). An example of the fit of the $4883 \AA$ line for star 14080 is given in Fig. 2.

Zr II: the line at $5112 \AA$ is the only clean usable line included in our spectral range. We adopted the $g f$ and excitation potential listed by Biemont et al. (1981).

Ba II: four lines were used to estimate the abundance: 4934.1, 5853.7, 6141.7, and 6496.9 А. We used spectral synthesis for the bluest line, since it is affected by considerable blending with a strong Fe line in the spectra under consideration. For the remaining lines, the abundance was measured through their $E W \mathrm{~s}$, because they are clean from blending for the stars of NGC 1851. The transition parameters adopted are from Sneden et al. (1996). An example of the fit of the $5853 \AA$ line for star 14080 is given in Fig. 2. Even though we derived the abundance from $E W$ s for this particular line, we chose it to illustrate that the Ba abundance from V10 for this particular star is not a good fit to our data (at least using our adopted atmospheric parameters). We note that abundances derived from both a synthesis and $E W$ agree very well for the plotted line.

La II: we used four lines (4921.0, 4921.8, 5114.5, and $6262.3 \AA$ ). The three bluest ones were synthesized, as they have a non-negligible hyperfine structure. Abundances for the other lines were derived from $E W$ measurements. The transition parameters adopted are from Sneden et al. (2003). An example of the fit to the $5114.5 \AA$ line for star 14080 is given in Fig. 2.

Ce II: we used one line at $5274.3 \AA$. The transition parameters adopted are from Sneden et al. (2003). We assign a smaller weight to our Ce abundances, because of possible blending of this line.

Nd II: we used nine lines for which we measured $E W \mathrm{~s}$ (5092.8, 5130.6, 5165.1, 5212.4, 5234.2, 5249.6, 5293.2, 5311.5, and $5319.8 \AA$ ). The transition parameters adopted are from Sneden et al. (2003). 
Table 9. Mean abundances from UVES and GIRAFFE.

\begin{tabular}{|c|c|c|}
\hline \multirow[t]{2}{*}{ Element } & UVES & GIRAFFE \\
\hline & $n$ avg rms & $n$ avg rms \\
\hline$[\mathrm{O} / \mathrm{Fe}]_{\mathrm{I}}$ & $13+0.040 .25$ & $86-0.010 .21$ \\
\hline$[\mathrm{Na} / \mathrm{Fe}]_{\mathrm{I}}$ & $13+0.260 .25$ & $120+0.190 .28$ \\
\hline$[\mathrm{Mg} / \mathrm{Fe}]_{\mathrm{I}}$ & $13+0.350 .03$ & $119+0.380 .04$ \\
\hline$[\mathrm{Al} / \mathrm{Fe}]_{\mathrm{I}}$ & $13+0.130 .21$ & \\
\hline$[\mathrm{Si} / \mathrm{Fe}]_{\mathrm{I}}$ & $13+0.380 .03$ & $120+0.390 .03$ \\
\hline$[\mathrm{Ca} / \mathrm{Fe}]_{\mathrm{I}}$ & $13+0.300 .02$ & $121+0.330 .04$ \\
\hline$[\mathrm{Sc} / \mathrm{Fe}] \mathrm{II}$ & $13-0.010 .08$ & $121+0.030 .06$ \\
\hline$[\mathrm{Ti} / \mathrm{Fe}]_{\mathbf{I}}$ & $13+0.170 .07$ & $118+0.150 .05$ \\
\hline$[\mathrm{Ti} / \mathrm{Fe}]_{\mathrm{II}}$ & $13+0.170 .05$ & \\
\hline$[\mathrm{V} / \mathrm{Fe}]_{\mathrm{I}}$ & $13-0.060 .09$ & $116-0.130 .12$ \\
\hline$[\mathrm{Cr} / \mathrm{Fe}]_{\mathrm{I}}$ & $13-0.020 .06$ & $113+0.050 .11$ \\
\hline$[\mathrm{Mn} / \mathrm{Fe}]_{\mathrm{I}}$ & $13-0.340 .08$ & \\
\hline$[\mathrm{Fe} / \mathrm{H}]_{\mathrm{I}}$ & $13-1.180 .07$ & $121-1.160 .05$ \\
\hline$[\mathrm{Fe} / \mathrm{H}]_{\mathrm{II}}$ & $13-1.140 .12$ & $101-1.130 .07$ \\
\hline$[\mathrm{Co} / \mathrm{Fe}] \mathrm{I}$ & $13-0.240 .06$ & $49-0.030 .07$ \\
\hline$[\mathrm{Ni} / \mathrm{Fe}] \mathrm{I}$ & $13-0.100 .03$ & $120+0.020 .07$ \\
\hline$[\mathrm{Cu} / \mathrm{Fe}] \mathrm{I}$ & $13-0.460 .07$ & \\
\hline$[\mathrm{Y} / \mathrm{Fe}]_{\mathrm{II}}$ & $13+0.270 .15$ & \\
\hline$[\mathrm{Zr} / \mathrm{Fe}] \mathrm{II}$ & $13+0.260 .11$ & \\
\hline$[\mathrm{Ba} / \mathrm{Fe}]_{\mathrm{II}}$ & $13+0.480 .26$ & $101+0.490 .22$ \\
\hline$[\mathrm{La} / \mathrm{Fe}]_{\mathrm{II}}$ & $13+0.230 .17$ & \\
\hline$[\mathrm{Ce} / \mathrm{Fe}]_{\mathrm{II}}$ & $13+0.690 .20$ & \\
\hline$[\mathrm{Nd} / \mathrm{Fe}]_{\mathrm{II}}$ & $12+0.670 .15$ & \\
\hline$[\mathrm{Eu} / \mathrm{Fe}]_{\mathrm{II}}$ & $13+0.670 .11$ & \\
\hline$[\mathrm{Dy} / \mathrm{Fe}]_{\mathrm{II}}$ & $13+0.670 .12$ & \\
\hline
\end{tabular}

Eu II: we synthesised two spectral lines, at 6437.6 and $6645.1 \AA$. This technique was used because they are affected by considerable hyperfine splitting. The transition parameters adopted are from Sneden et al. (2003). An example of the fit to the $6645 \AA$ line for star 14080 is given in Fig. 2.

Dy II: an abundance was derived from the $E W$ of the line at $5169.7 \AA$. The transition parameters adopted are from Sneden et al. (2003).

Results are given in Tables 7 and 8 for stars with UVES and GIRAFFE spectra, respectively. The averages of all measured elements with their rms scatter are listed in Table 9.

\section{Metallicity spread and stellar population components}

\subsection{Metallicity spread in NGC 1851}

In Carretta et al. (2010c), we argued that we detected a small but real metallicity spread in NGC 1851 by comparing observed abundances with internal errors. We deem that our results are robust because thy are based on a statistically significant sample. NGC 1851 shows a much larger dispersion in $[\mathrm{Fe} / \mathrm{H}]$ than other GCs of similar metal abundance, when examined with the same instrumentation and technique of analysis (M4 and M5: see Fig. 3).

Apart from there being a larger scatter among the brighter stars observed with UVES (discussed in detail in Carretta et al. 2009c), the iron dispersion observed in M 4 and M 5 is compatible with internal errors associated with the analysis, 0.025 and $0.023 \mathrm{dex}$, respectively. However, if we try to fit the iron distribution in NGC 1851 using a Gaussian with $F W H M$ equal to 0.024 dex (the average error from M 4 and M 5, Fig. 4) the plot clearly indicates that there is a small intrinsic dispersion

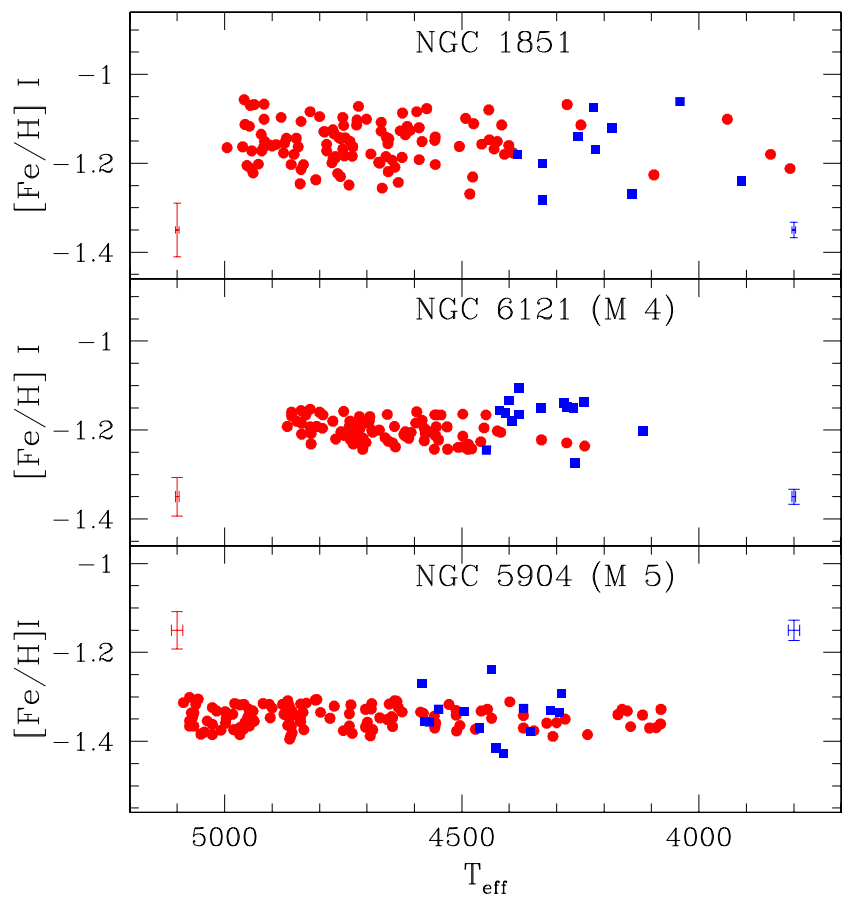

Fig. 3. $[\mathrm{Fe} / \mathrm{H}]$ ratios as a function of effective temperature for stars analysed in NGC 1851 (upper panel), M 4 (middle panel), and M 5 (lower panel), both from Carretta et al. (2009a,b). The filled red circles indicate stars with GIRAFFE spectra, the blue filled squares stars with UVES spectra. Error bars in the left and right side of the panels refer respectively to our GIRAFFE and UVES data and indicate star-to-star errors.

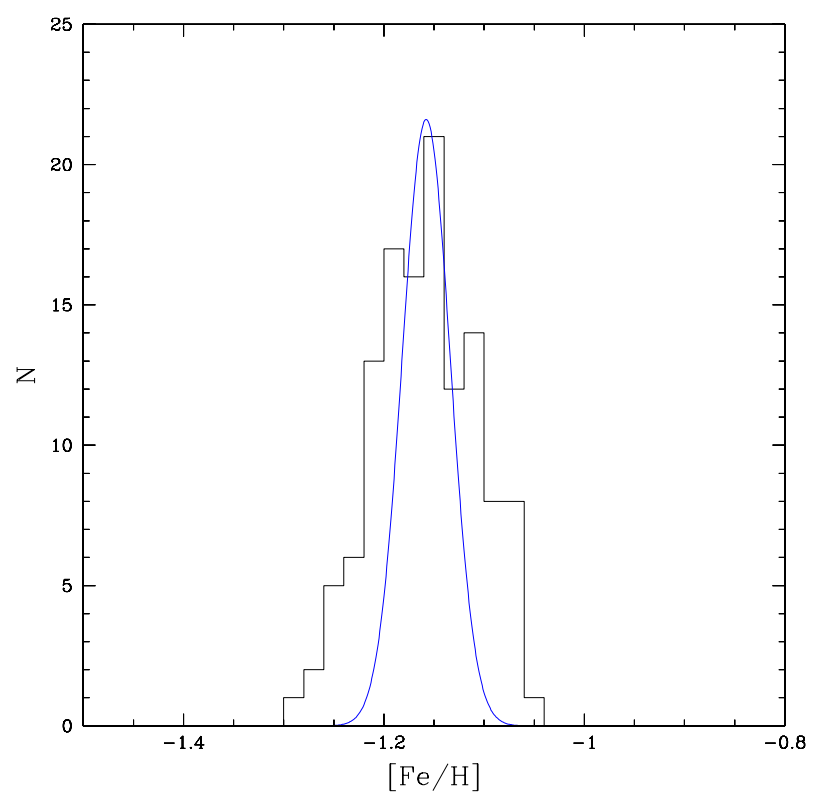

Fig. 4. Histogram of $[\mathrm{Fe} / \mathrm{H}]$ ratios for our NGC 1851 stars. For the gaussian curve we adopted as $F W H M=0.024$, that is the average standard deviation in $[\mathrm{Fe} / \mathrm{H}]$ between the mono-metallic GCs M $4(\mathrm{rms}=0.025)$ and M $5(\mathrm{rms}=0.023$, see Carretta et al. 2009c).

in the iron content that cannot be accounted for by internal errors alone.

On the other hand, the comparison of the scatter in Fe abundances (Table 9) with the internal errors estimated in the 
Appendix shows that the observed dispersion in $[\mathrm{Fe} / \mathrm{H}]$ I for NGC 1851 is statistically significant for stars with metallicity from UVES, as pointed out in Carretta et al. (2010c). In this case, internal errors are of the order of 0.03 dex, much lower than the observed scatter $(0.07 \mathrm{dex})$. The case is less compelling for the abundances from GIRAFFE data, for which we obtained an internal error in $[\mathrm{Fe} / \mathrm{H}](0.043 \mathrm{dex})$ not much lower than the observed scatter $(0.051 \pm 0.005 \mathrm{dex})$. However, putting together the highly significant result obtained from UVES spectra with the weaker evidence coming from GIRAFFE ones and the consideration that the observed scatter is much larger than for the similar clusters M4 and M5, we conclude that there is a real dispersion in $[\mathrm{Fe} / \mathrm{H}]$ values within NGC 1851.

A similar amount of intrinsic scatter was also found by Yong $\&$ Grundahl (2008). The eight stars observed in that study have an average $[\mathrm{Fe} / \mathrm{H}]=-1.268 \pm 0.030$ with a $1 \sigma$ rms scatter of 0.084 dex. Although there is a small difference between this value and that of the present analysis (most likely caused by different temperature and $g f$ scales adopted), their study agrees with us about the reality of a small metallicity dispersion in NGC 1851. On the other hand, V10 did not find any scatter in $[\mathrm{Fe} / \mathrm{H}]$ among their 15 stars.

Given the method used to derive atmospheric parameters for individual stars, a potential contamination by AGB stars in our sample could increase the observed scatter. This might occur in particular for the UVES sample, where most of the stars are brighter than the magnitude limit at which it is easy to discriminate AGB from RGB stars (see Fig. 1). In this diagram, the AGB is clearly discernible from the RGB below $V \sim 14.5$ ( $T_{\text {eff }} \sim 4300 \mathrm{~K}$ and $M_{V} \sim-1$ ). For fainter stars, we simply omitted AGB stars from our sample. For brighter stars, the two sequences do not appear to be any more distinct, because these bright AGB stars are $<50 \mathrm{~K}$ warmer than first ascent RGB stars (this value is derived from the isochrone by Bertelli et al. 2008 at $Z=0.001$ and $12.6 \mathrm{Gyr}$ ). Our sample may then include some bright AGB stars. However, we believe that this is not a source of concern for several reasons. First, we expect very few AGB stars, since they represent less than about $20 \%$ of the red giants: hence, among the 17 observed stars brighter than the limit we expect only about three AGB interlopers. Second, the errors produced in the analysis are small. Even if we make an error of $0.2 M_{\odot}$, by mistaking AGB stars for RGB stars, this translates into an error of 0.1 dex in $\log g$, when all the other parameters are held fixed; and, as mentioned above, the errors made in the $T_{\text {eff }}$ values are $<50 \mathrm{~K}$. Using the sensitivities listed in Tables A.2 and A.1 in the Appendix, the gravity error would imply errors in the derived $[\mathrm{Fe} / \mathrm{H}]$ ratio of $-0.003 \mathrm{dex}$ and +0.012 dex for GIRAFFE and UVES, respectively. Owing to the errors in temperature, bright AGBs confused in the RGB sample could appear up to be 0.04-0.06 dex more metal-poor than the other stars. These effects are very small. Furthermore, we note that the observed metallicity scatter is almost constant over the whole temperature range, regardless of whether we include the temperature range where possible contamination by AGB stars exist (see Fig. 3).

Therefore, the observed spread seems real and not to be caused by a neglected contamination from AGB stars. This is corroborated by the good agreement we obtain, on average, between abundances based on neutral and singly ionised species (e.g. iron) that supports the atmospheric parameters we used, including surface gravities $\log g$. More generally, we do not attribute much weight to the small offset between the abundances of neutral and singly ionised iron, which is well within $1 \sigma \mathrm{rms}$ scatter, thus hardly significant. The $[\mathrm{Fe} / \mathrm{H}]$ II abundances rest on only one-two lines for GIRAFFE spectra and bright stars (those observed with UVES) are also cooler, on average, hence more affected by possible NLTE effects. In all cases, we do not use $[\mathrm{Fe} / \mathrm{H}]$ II in any of our conclusions.

Finally, we stress that in the present analysis the observed spreads are only lower limits, owing to the particular way we derive the final temperatures using a mean relation between $T_{\text {eff }}(V-K)$ and the $K$ magnitude along the RGB. While this technique allows us to have extremely small internal errors (see Carretta et al. 2007a, for details), it also tends to slightly reduce any real metallicity spread along the RGB, leading us to adopt an effective temperature corresponding to a mean ridge line. Had we iteratively used slightly different temperaturemagnitude relations for each component, we would have obtained quite similar results, on average, the maximum additional difference in $[\mathrm{Fe} / \mathrm{H}]$ amounting to less than 0.04 dex.

In addition to these results, the most striking evidence of a difference in the stellar populations on the RGB in NGC 1851 is the spatial distribution of the metal-rich (MR) and metal-poor (MP) components (Carretta et al. 2010c). We found that there is a clearcut evidence that stars more metal-poor than the cluster average $([\mathrm{Fe} / \mathrm{H}]=-1.16)$ are more centrally concentrated than more metal-rich stars, with a very high degree of confidence. There is something intrinsically different between the MR and MP populations in NGC 1851.

This finding bypasses any debate on the reality of the radial distributions of SGB stars in the existing literature and was possible only owing to the large number of stars analysed in a very homogeneous way. This observation stimulated the working hypothesis proposed in Carretta et al. (2010c) that NGC 1851 might be the result of a merger of two originally distinct clusters, of slightly different metallicities.

There are other differences between the MR and MP components, besides those in $[\mathrm{Fe} / \mathrm{H}]$ and radial distribution, in the abundances of $\alpha$-capture and neutron-capture elements (see below). Moreover, in each individual component there is a welldeveloped Na-O anticorrelation (Carretta et al. 2010c), which is the chief signature of a genuine GC according to Carretta et al. (2010a). A very similar finding has been obtained for M 22 (Marino et al. 2011), which has several analogies with NGC 1851.

\subsection{A cluster analysis approach to stellar components in NGC 1851}

While the choice of the average $[\mathrm{Fe} / \mathrm{H}]$ as a separating value between the two populations is the simplest one, it is also arbitrary. To seek a more objective criterion, we exploit the main results obtained up to now for NGC 1851 (Carretta et al. 2010c): (i) there is a real (small) metallicity dispersion in the cluster; where (ii) the MP component is more centrally concentrated; and moreover (iii) the MR component contains a larger amount of $s$-process elements. These observations suggest to use the run of $[\mathrm{Fe} / \mathrm{H}]$ as a function of $[\mathrm{Ba} / \mathrm{H}]$ to help us separate the two stellar components in NGC 1851. For this data, we performed a cluster analysis using the $k$-means algorithm (Steinhaus 1956; MacQueen 1967), as implemented in the $R$ statistical package (R Development Core Team 2011), a system for statistical computation and graphics, freely available on-line ${ }^{7}$. Two key features of $k$-means are:

- Euclidean distance is used as a metric and variance is used as a measure of cluster scatter. The various parameters used

${ }^{7}$ http://www.R-project.org 


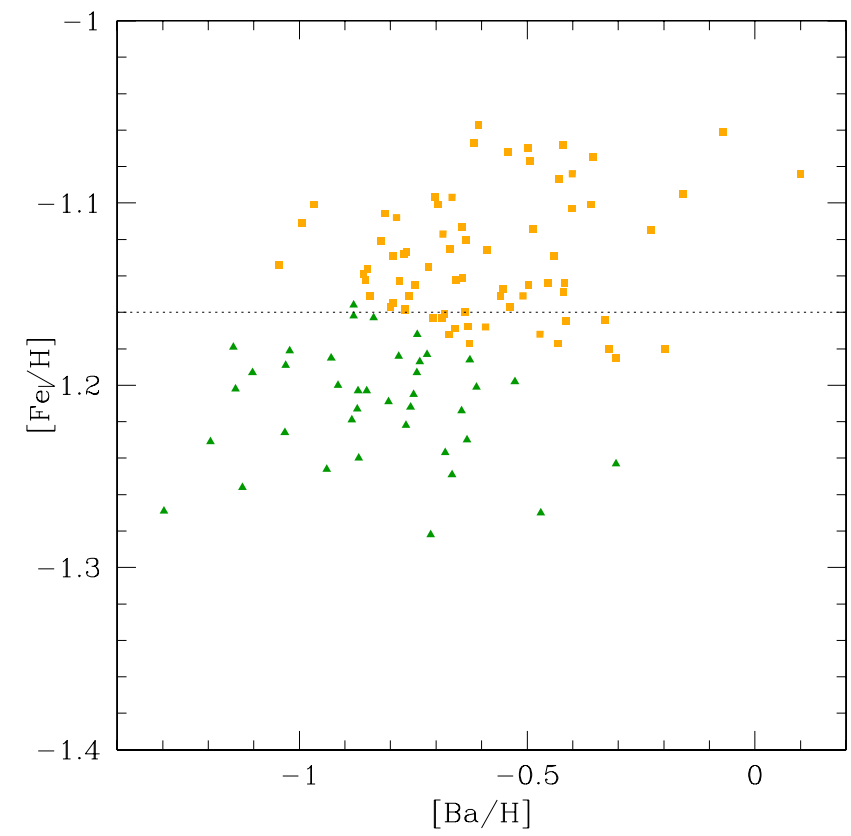

Fig. 5. Diagnostic diagram $[\mathrm{Fe} / \mathrm{H}]$ vs. $[\mathrm{Ba} / \mathrm{H}]$ for the stellar components in NGC 1851. The different symbols and colour coding show the results of the cluster analysis using $\mathrm{Fe}$ and $\mathrm{Ba}$ abundance as parameters (see text). Stars indicated with orange filled squares are attributed to the MR component; stars indicated with green filled triangles are assigned to the MP component. The dotted line indicates the average value $[\mathrm{Fe} / \mathrm{H}]=-1.16 \mathrm{dex}$ for NGC 1851 that we previously adopted as a separating value.

should be transformed before computing these distances, in order to weight them adequately. A sensible choice is to normalize them to the observational errors. In practice, as normalization factors we assumed errors of 0.04 and 0.20 dex for $[\mathrm{Fe} / \mathrm{H}]$ and $[\mathrm{Ba} / \mathrm{H}]$, respectively.

- The number of clusters $k$ is an input parameter, and an inappropriate choice of $k$ may yield poor results. Given that the ratio of observed scatter of data to observational errors is not large, we adopted $k=2$. There may of course be more than two groups of stars in NGC 1851, but we wished to limit the number of hypotheses at this stage.

A key limitation of $k$-means is its cluster model. The concept is based on spherical clusters that are separable in such a way that the mean value converges towards the cluster centre. The clusters are expected to have similar populations, so that the assignment to the nearest cluster centre is the correct assignment. When the clusters have very different sizes, this may result in an inaccurate assignement of members to clusters. However, in the present case, we expect that most of the scatter within one cluster is due to observational errors, which are similar for the different clusters. We then considered whether the assumption of similar size for the different clusters is acceptable. This does not mean, of course, that the occasional assignment of some member (that is, a star) to a particular cluster is questionable.

The results are shown in Fig. 5, where the two components are indicated with different symbols and colours. We also superimpose a line indicating the average value $[\mathrm{Fe} / \mathrm{H}]=-1.16 \mathrm{dex}$ for NGC 1851, adopted by Carretta et al. (2010c) to separate the two components using only the metallicity.

We note that 13 stars do not have $\mathrm{Ba}$ abundances because UVES or HR13 GIRAFFE spectra are unavailable for these stars. For 10 stars, it is easy to unambiguously assign

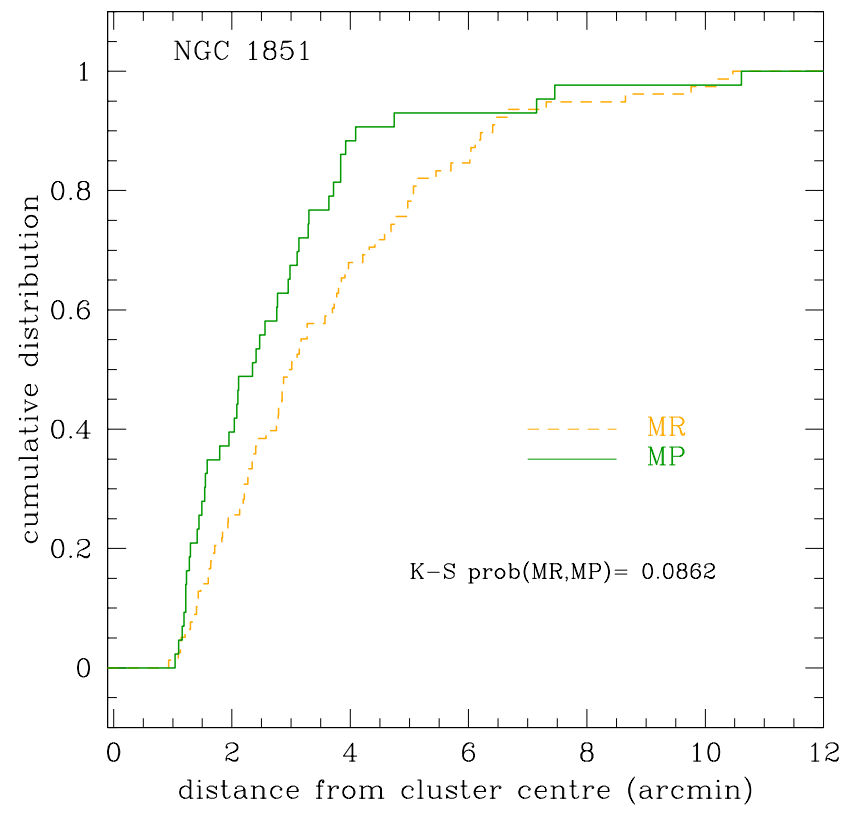

Fig. 6. Cumulative distribution of radial distances for all stars in our sample, divided into MP (green solid line) and MR (dashed orange line) components.

a population simply according to the metallicity (most of them falling above $[\mathrm{Fe} / \mathrm{H}]=-1.10$ or below $[\mathrm{Fe} / \mathrm{H}]=-1.20 \mathrm{dex}$ ). However, for three stars $(28116,33385,40300)$ this is not possible and their status is currently uncertain.

The cluster analysis shows that the new components are similar to, but not exactly coincident with, those defined in Carretta et al. (2010c). The separation line is slightly slanted and is not simply an average value, when accounting also for the enhanced level of $\mathrm{Ba}$ in the most metal-rich component. However, for simplicity, we continue to refer to both the MR and MP components. The main difference is that the fraction of stars in the MP component decreases from $50 \%$ to $36 \%$, while the MR one increases to $64 \%$. These fractions are tantalisingly close to the fractions of stars observed on the one hand on the faint SGB (45\%) and the BHB (40\%), and on the other hand on the bright SGB $(55 \%)$ and RHB (60\%, see Milone et al. 2008). This might suggest a tentative link according to the new population ratios, although this link should actually be confirmed by determining the abundances of the SGB and HB stars.

Also with this new definition the MP component is more concentrated than the MR one to a reasonable level of confidence (see Fig. 6).

Milone et al. (2009) considered the radial distributions of the different populations in NGC 1851, claiming that there is no difference between the two SGBs. Their sample size is far larger than ours, and since their conclusion is apparently different, we re-examined their result. In their analysis, they considered three possible cases (labelled A-C), depending on the interpretation of the splitting of the SGB. Case A represents a simple age difference (about $1 \mathrm{Gyr}$ ); case B a combination of age difference (about 2 Gyr) and CNO abundance variations; and case $\mathrm{C}$ a difference only in the sum of CNO abundances. If we use as metrics for the comparison the Pearson correlation coefficient between the radius and the fraction of faint SGB stars, there is a slightly more than $5 \%$ probability that the correlation is random for cases $\mathrm{A}$ and $\mathrm{B}$, while there is no clear difference for case $\mathrm{C}$. Since they seems to prefer this last scenario, Milone et al. (2009) concluded that there is no difference in the radial distribution of 
the different populations of NGC 1851. However, much more could be said apropos. First, obviously a lack of evidence is not evidence of a lack. Second, in their data there is indeed some hint of a radial gradient, the faint SGB being more concentrated than the bright SGB. While the test considered above does not seem strong enough to allow a conclusion at a high confidence level, other tests such as the Kolmogorov-Smirnov one are more powerful in revealing differences among distributions, and would seem more appropriate in this context. While we have not at our disposal enough data to perform the Kolmogorov-Smirnov test on their data, we might use other metrics. For instance, we may compare the weighted averages of the three innermost and the three outermost bins. Using this approach, we found differences of $0.11 \pm 0.06,0.17 \pm 0.07$, and $0.06 \pm 0.06$ for cases $\mathrm{A}-\mathrm{C}$. Summarizing, the lack of evidence claimed by Milone et al. (2009) is related to the statistics they used in their analysis and to the interpretative scenario they favour for the separation of NGC 1851 into two populations. The set of statistics used is likely not the most powerful one, and there is no strong spectroscopic evidence of a variation in the sum of $\mathrm{CNO}$ abundances in NGC 1851 (see also V10). We therefore conclude that their data cannot be used to conclude that there is no difference in the radial distributions of the different populations of NGC 1851.

\subsection{Kinematics of stellar components in NGC 1851}

In principle, if the MP and MR components come from two distinct sub-units they could also have different kinematics, which would then be clearly evident in their velocity distribution. However, we found no significant differences: the velocity dispersion increases from the outskirts towards the cluster centre (see Fig. 7 and Table 10) but for the MR and MP the pattern is similar, apart from the different concentration of stars in the two components.

Given the limitations of our sample, we cannot tell whether this is due to insufficient data or has some real physical meaning. We only note that if the merger is recent, there might be only a small dynamical effect, because otherwise after the merging the cluster would not be relaxed, and there might be no energy equipartition between the different populations. The merging between the two putative clusters could well be an event that occurred only a few Gyr ago: the relaxation time at the half mass radius (and we sampled a region external to this radius) is about 0.7 Gyr (Harris 1996), and a full cluster relaxation does require some 2-3 relaxation times. In the framework outlined in Carretta et al. (2010a) for the formation model of GCs, the only constraint is that the merger between the two clusters must have occurred before their host dSph galaxy merged with our Galaxy. However, we do not know when this possible interaction happened. If some debris of this putative dSph still exist (as suggested by several authors, e.g. Carballo-Bello et al. 2010; Olszewski et al. 2009) then the event could be rather recent. More data and detailed dynamical modelling would be required; however, this is beyond the scope of the present work, which is focused on the chemical tagging.

\section{The complex nucleosynthesis in NGC $\mathbf{1 8 5 1}$}

\subsection{Nucleosynthesis in the globular cluster environment: the proton-capture elements}

The pattern of observed (anti)correlations among light elements such as $\mathrm{C}, \mathrm{N}, \mathrm{O}, \mathrm{F}, \mathrm{Na}, \mathrm{Mg}, \mathrm{Al}$, and $\mathrm{Si}$ requires a specific chain of events, restricted to the proto-cluster environment

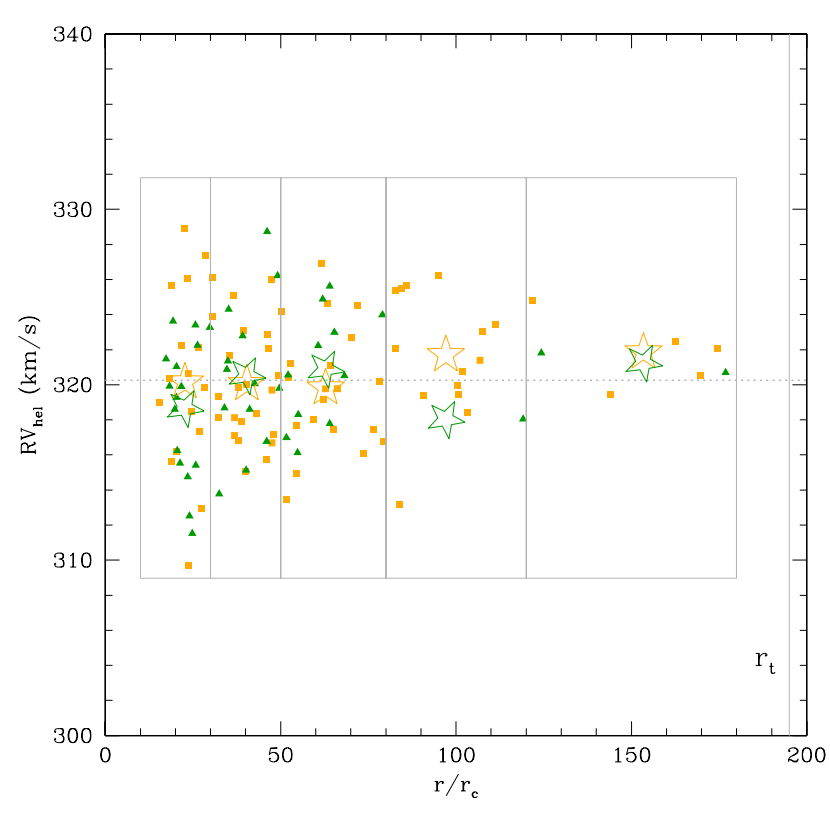

Fig. 7. Distribution of radial velocities for all stars in our sample, divided into MP (filled greed triangles) and MR (filled orange squares) components. The $x$ axis shows the distance from the cluster centre (in units of the core radius $r_{\mathrm{c}}$ ). The larger symbols, also in green and orange, represent the average RV of the two sub-samples, computed in the boxes indicated in the figure. The dotted horizontal line represents the global average of the sample $\left(320.26 \mathrm{~km} \mathrm{~s}^{-1}\right.$, with a rms of 3.74) and the vertical line indicates the tidal radius. The average RVs at the various distances are given in Table 10.

Table 10. Average RVs for MP and MR stars at different distances from the cluster centre.

\begin{tabular}{lcccccc}
\hline \hline $\begin{array}{l}\text { Dist. } \\
\left.\text { (in } r_{\mathrm{c}}\right)\end{array}$ & $\begin{array}{c}\langle\mathrm{RV}(\mathrm{MR})\rangle \\
\left(\mathrm{km} \mathrm{s}^{-1}\right)\end{array}$ & rms & $\begin{array}{c}\text { Nr. } \\
\text { MR }\end{array}$ & $\begin{array}{c}\langle\mathrm{RV}(\mathrm{MP})\rangle \\
\left(\mathrm{km} \mathrm{s}^{-1}\right)\end{array}$ & rms & $\begin{array}{c}\text { Nr. } \\
\mathrm{MP}\end{array}$ \\
\hline $10-30$ & 320.16 & 5.25 & 16 & 318.67 & 3.90 & 16 \\
$30-50$ & 320.05 & 3.26 & 23 & 320.55 & 4.25 & 13 \\
$50-80$ & 319.82 & 3.51 & 20 & 320.91 & 3.30 & 11 \\
$80-120$ & 321.70 & 3.59 & 14 & 318.04 & 0.00 & 1 \\
$>120$ & 321.86 & 2.05 & 5 & 321.25 & 0.78 & 2 \\
\hline
\end{tabular}

(see e.g. Gratton et al. 2004). An unavoidable requirement for a $\mathrm{GC}$ is the presence of a first generation of stars providing at their death the material, processed through proton-capture reaction in $\mathrm{H}$-burning at high temperature, that participated in the formation of the second stellar generation(s) with a modified chemical composition.

These considerations, together with the existence of the $\mathrm{Na}-\mathrm{O}$ anticorrelation among stars of each metallicity component on the RGB of NGC 1851, support the merger of two clusters as a viable origin of the observed characteristics of this cluster. We now examine whether this simple working hypothesis is unaffected by our revised classification (Sect. 4.2) of stellar populations and the full set of abundances derived for NGC 1851.

The (anti)correlations among proton-capture elements in NGC 1851 are summarised in Fig. 8 for stars with UVES spectra, from which the whole set of $\mathrm{Na}, \mathrm{O}, \mathrm{Mg}$, and $\mathrm{Al}$ abundances is available. NGC 1851 shows a moderate Na-O anticorrelation, and a well-defined $\mathrm{Na}-\mathrm{Al}$ correlation, with a not extremely high production of Al. In Fig. 8, there is a hint that stars of the 

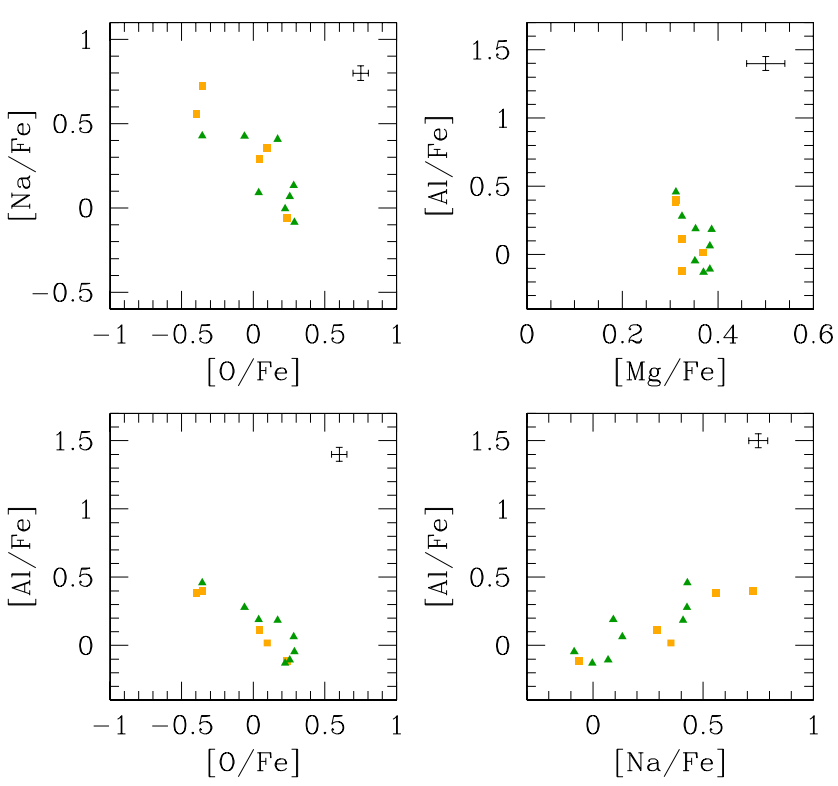

Fig. 8. Na-O (top-left panel), $\mathrm{Mg}-\mathrm{Al}$ (top-right panel), $\mathrm{Al}-\mathrm{O}$ (bottomleft panel) anticorrelation, and $\mathrm{Na}-\mathrm{Al}$ correlation (bottom-right panel) for stars with UVES spectra in NGC 1851. Green triangles and orange squares indicate stars of the MP and MR component, respectively. Error bars are star-to-star errors.

MR component may reach more extreme processing than stars of the MP component, but this sample is limited.

The global Na-O anticorrelation is formed by stars of a wide range of metallicities, and its extension (as measured by the interquartile range $\mathrm{IQR}[\mathrm{O} / \mathrm{Na}]=0.693$ ) closely follows the strong correlations with the total cluster mass (using the absolute visual magnitude $M_{V}=-8.33$ as a proxy) and with the maximum temperature along the HB derived and widely discussed in Carretta et al. (2010a) (see Fig. 9) ${ }^{8}$.

The cluster analysis, based only on $\mathrm{Fe}$ and $\mathrm{Ba}$ abundances, separates stars of NGC 1851 into two sub-groups, each one following a clean $\mathrm{Na}-\mathrm{O}$ anticorrelation. The anticorrelation is slightly more extended for the MR component $(\mathrm{IQR}[\mathrm{O} / \mathrm{Na}]=0.750)$ than for the MP one $(\mathrm{IQR}[\mathrm{O} / \mathrm{Na}]=0.674)$ (see Fig. 10). As an exercise, we tentatively distributed the total $M_{V}=-8.33$ of NGC 1851 over the two MR and MP components by using the number of the RHB (191) and of BHB stars (116), respectively, adopted by Gratton et al. (2010b) in their study of the $\mathrm{HB}$ in this and other GCs. The resulting values are $M_{V}=$ -7.81 and $M_{V}=-7.27$ for the MR and MP groups, respectively. When coupled with the IQR values derived above, the two components of NGC 1851 nicely fit the overall relation between $M_{V}$ and IQR[O/Na] (green and orange symbols in the left panel of Fig. 9). This supports the idea that the two components in NGC 1851, selected using independent parameters (Fe, $\mathrm{Ba}$ ), do behave as two individual GCs with slightly different total masses.

To supplement this evidence, we used the Strömgren photometry by Grundahl (available through the database used by Calamida et al. 2007) to plot the stars of the P, I, and E components in NGC 1851. The definition of the primordial (P), intermediate (I), and extreme (E) population in a cluster, based on

\footnotetext{
8 Note that the analysis considered here for $\omega$ Cen (Johnson \& Pilachowski 2010) is not strictly consistent with the other determinations shown in the plot, which come from our FLAMES survey.
}

$\mathrm{Na}$ and $\mathrm{O}$ abundances, is illustrated in Carretta et al. (2009a), where we showed how the P (Na-poor, O-rich) stars of the first generation lie on a narrow strip to the blue of the RGB, while the $\mathrm{I}+\mathrm{E}$ second generation stars are spread out to the red in a Strömgren $u, u-b$ CMD. The $u, u-b$ CMD is a good choice of plane to search for this segregation, since any division is due to the effect of the absorption by $\mathrm{NH}$ in the wavelength range sampled by near-UV bandpasses such as the Johnson $U$ (e.g. Marino et al. 2008), the Strömgren $u$, or even the Sloan $u$ bandpass (see Lardo et al. 2011), as also discussed by Sbordone et al. (2011) and Carretta et al. (2011). We show in Fig. 11 the results for NGC 1851 using stars observed both by us and Grundahl: in each metallicity group, the three components are nicely distributed along the RGB as if each group were an individual GC.

The fraction of stars in the primordial, intermediate, and extreme components for NGC 1851 and the two MR and MP metallicity components are listed in Table 11. Even considering the rather large errors from the Poisson statistics, the fraction of primordial stars in the MR component appears to be smaller than the $\mathrm{P}$ fraction in the MP component. These fractions are computed using stars with both $\mathrm{O}$ and $\mathrm{Na}$ abundances (Carretta et al. 2009a). If we use only $\mathrm{Na}$ abundances, regardless of $\mathrm{O}$ abundance (this by definition allows us only to separate first and second generation stars, since it is a criterion "blind" to the separation between I and E stars) then the fractions of primordial stars in the MR and MP components are still rather different: $21 \pm 5 \%$ and $40 \pm 10 \%$, respectively.

Figure 12 shows the location of a slight change in the mean values of $\mathrm{O}$ and $\mathrm{Na}$ abundances, just at the level of the bump on the RGB. We remind the reader that our interpretation (that the change is caused by the mix of first and second generation stars, each generation having its own slightly different He content, see Salaris et al. 2006; and Bragaglia et al. 2010) is based on the observation that the ratios $[\mathrm{Na} / \mathrm{Fe}]$ and $[\mathrm{O} / \mathrm{Fe}]$ run essentially flat as a function of luminosity in field stars (Gratton et al. 2000). While lighter species (such as Li, C, and N) can be mixed up, the $\mathrm{ON}$ and $\mathrm{NeNa}$ cycles require higher temperatures: they are cycled in the inner layers of the H-burning shell that cannot be reached by the extra-mixing processes even after the RGB-bump. In the case of NGC 1851, where probably we could see the result of two distinct clusters, each with its own Na-O anticorrelation and P, I, and E components, we expect an additional smearing of the bump in the RGB luminosity function.

Finally, we considered the other elements participating in the proton-capture reactions, namely $\mathrm{Mg}$ and $\mathrm{Si}$, using the large sample of stars for which we measured homogeneous abundances from GIRAFFE spectra. In NGC 1851 (Fig. 13), the abundances of $\mathrm{Mg}$ are depleted as $\mathrm{O}$ is depleted and $\mathrm{Na}$ is enhanced, while the opposite happens for Si. The last result is probably due to the leakage from the $\mathrm{Mg}-\mathrm{Al}$ cycle on $\mathrm{Si}$, first discovered by Yong et al. (2005) in NGC 6752 and then found by Carretta et al. (2009b) in stars of several clusters, although only in the small samples of RGB stars with UVES spectra. This is the first time that these relations involving $\mathrm{Si}$ are observed in a very large sample of stars in an individual cluster. The large size of the sample (85 stars with $\mathrm{Mg}, \mathrm{Si}$, and $\mathrm{O}$; about 120 stars with $\mathrm{Mg}, \mathrm{Si}$, and $\mathrm{Na}$ ) ensure that all the (anti)correlations in Fig. 13 are robust to a high level of confidence. Although the internal errors are not negligible and the sizes of variations in the $[\mathrm{Si} / \mathrm{Fe}]$ abundance ratios are not dramatic (about $0.1-0.15 \mathrm{dex}$ ), these relations tell us that at least part of the material from which the second generation stars formed was processed at a temperature exceeding 

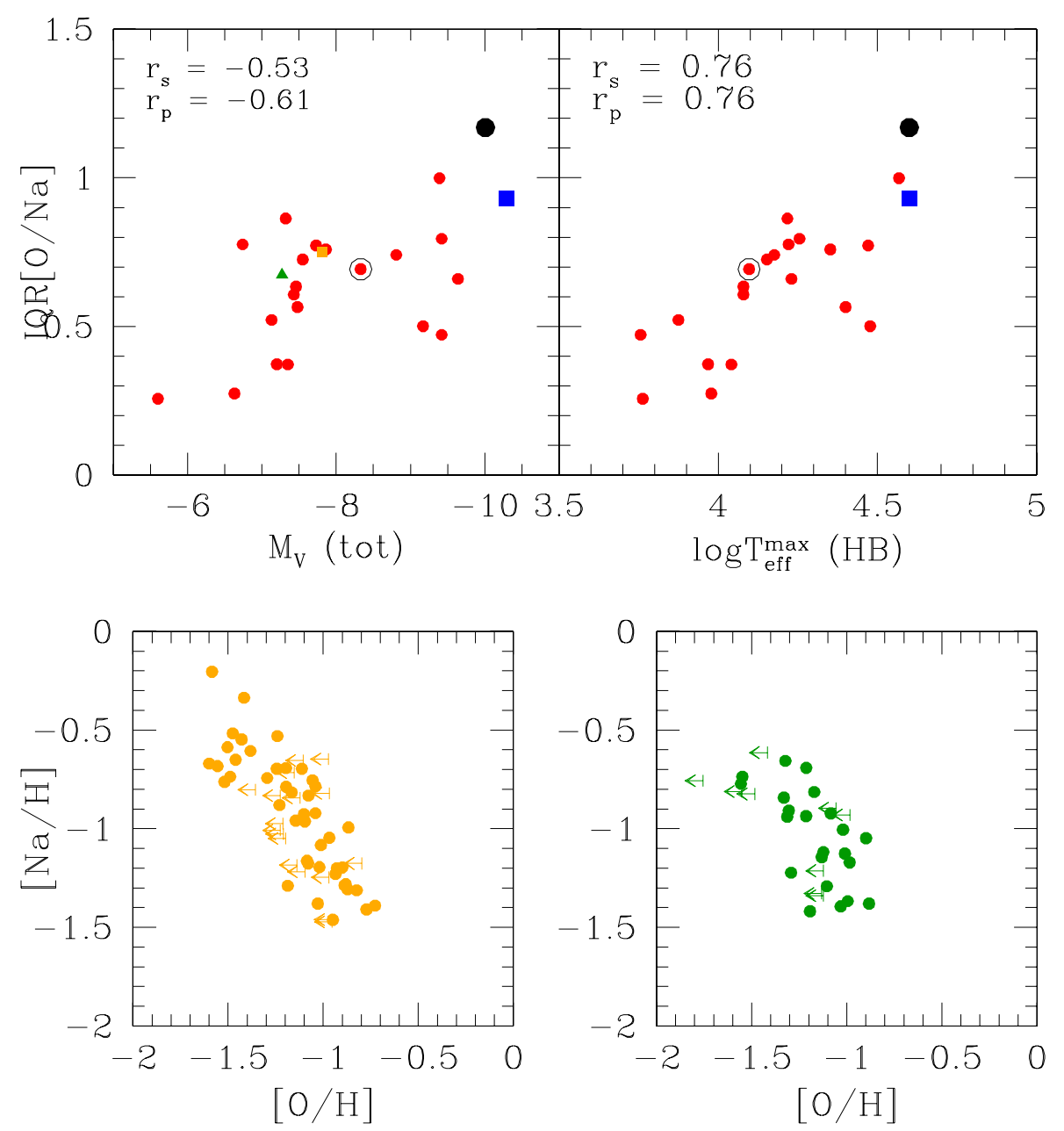

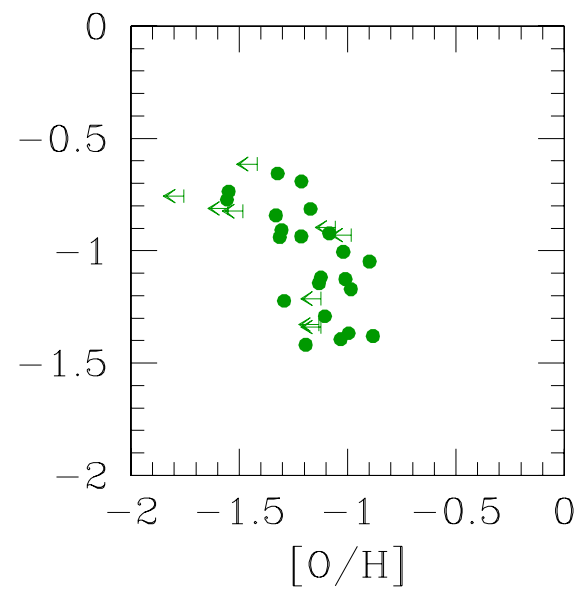

Fig. 9. Interquartile range of the $[\mathrm{O} / \mathrm{Na}]$ ratio, IQR $[\mathrm{O} / \mathrm{Na}]$, for the stars in NGC 1851 as a function of the total absolute visual magnitude (left panel) and of the maximum temperature reached by HB stars (mainly from Recio-Blanco et al. 2006, right panel). NGC 1851 is indicated by a encircled filled point. The large black filled circle and blue square are for M 54 and $\omega$ Cen, respectively. The IQR values come from Carretta et al. (2010b) and from data by Johnson \& Pilachowski (2010), respectively, and maximum temperatures estimated by Cassisi (2011, priv. comm.). The Spearman rank correlation coefficient $\left(r_{\mathrm{s}}\right)$ and the linear regression Pearson's correlation coefficient $\left(r_{\mathrm{p}}\right)$ are listed in each panel. In the left panel, the green triangle and the orange square indicate the values relative to the MP and MR components in NGC 1851 (see text).
Fig. 10. Na-O anticorrelation for stars of the MR (left panel) and of the MP (right panel) component in NGC 1851.

Table 11. Number of stars with both $\mathrm{Na}$ and $\mathrm{O}$ abundances and fraction of the primordial, intermediate, and extreme components.

\begin{tabular}{|c|c|c|c|c|}
\hline CASE & $\begin{array}{c}\text { N.stars } \\
(\mathrm{O}, \mathrm{Na}) \\
\text { GIR.+UVES }\end{array}$ & $\begin{array}{c}\text { Fraction } \\
\mathrm{P} \\
\text { component } \\
\end{array}$ & $\begin{array}{c}\text { Fraction } \\
\text { I } \\
\text { component }\end{array}$ & $\begin{array}{c}\text { Fraction } \\
\text { E } \\
\text { component }\end{array}$ \\
\hline NGC 1851 all & 95 & $31 \pm 6$ & $63 \pm 8$ & $6 \pm 3$ \\
\hline NGC $1851 \mathrm{MR}$ & 61 & $20 \pm 6$ & $72 \pm 11$ & $8 \pm 4$ \\
\hline NGC $1851 \mathrm{MP}$ & 34 & $44 \pm 11$ & $53 \pm 12$ & $3 \pm 3$ \\
\hline
\end{tabular}

$\sim 65$ million $\mathrm{K}$. This is the threshold for which the reaction producing ${ }^{28} \mathrm{Si}$ becomes effective in the $\mathrm{Mg}$ - $\mathrm{Al}$ cycle (Arnould et al. 1999).

Separating the sample into the MR and MP components, the relations of Fig. 13 still hold for each sub-group.

\subsection{Nucleosynthesis from massive stars: $\alpha$-elements}

The elements involved in $\alpha$-capture reaction are the main signature of nucleosynthesis in massive stars, which end their lives as core-collapse SNe. Here we consider of course the level of primordial enrichment of the gas from which the first stellar generation formed in the cluster. The abundance of some elements, such as $\mathrm{Mg}$, and $\mathrm{Si}$, may be subsequently modified by proton-capture reactions, as discussed in the previous section. No change should instead be observed for heavier elements, e.g. $\mathrm{Ca}$, between multiple generations in a GC. The chemical enrichment by massive stars received much interest after the proposal by Lee et al. (2009b) that chemical pollution by type II SNe might be discerned in GCs hosting multiple populations.

In Fig. 14, we show the run of $\alpha$-elements in NGC 1851 as a function of the temperature, using the large sample of stars with homogeneous abundances from GIRAFFE spectra. The mean overabundance is quite normal (Table 9) for cluster stars and the small observed scatter in $\mathrm{Mg}$ and $\mathrm{Si}$ is compatible with the $\mathrm{Mg}$ Al cycle producing only small changes in the primordial level of $\mathrm{Mg}$ and $\mathrm{Si}$, despite the noted anticorrelations and correlations discussed in the previous Sect. 5.1. No distinct trend is observed as a function of either effective temperature or metallicity, as is clearly evident from the adopted color coding.

However, the result is quite different if we plot the ratio with respect to the $\mathrm{H}$ abundance, as in Fig. 15: the absolute level of $\alpha$-elements is larger in the MR component of NGC 1851 than in the MP component. The evidence is somehow weaker in the case of $\mathrm{Ti}$ I but is very clear for $\mathrm{Mg}, \mathrm{Si}$, and $\mathrm{Ca}$. 

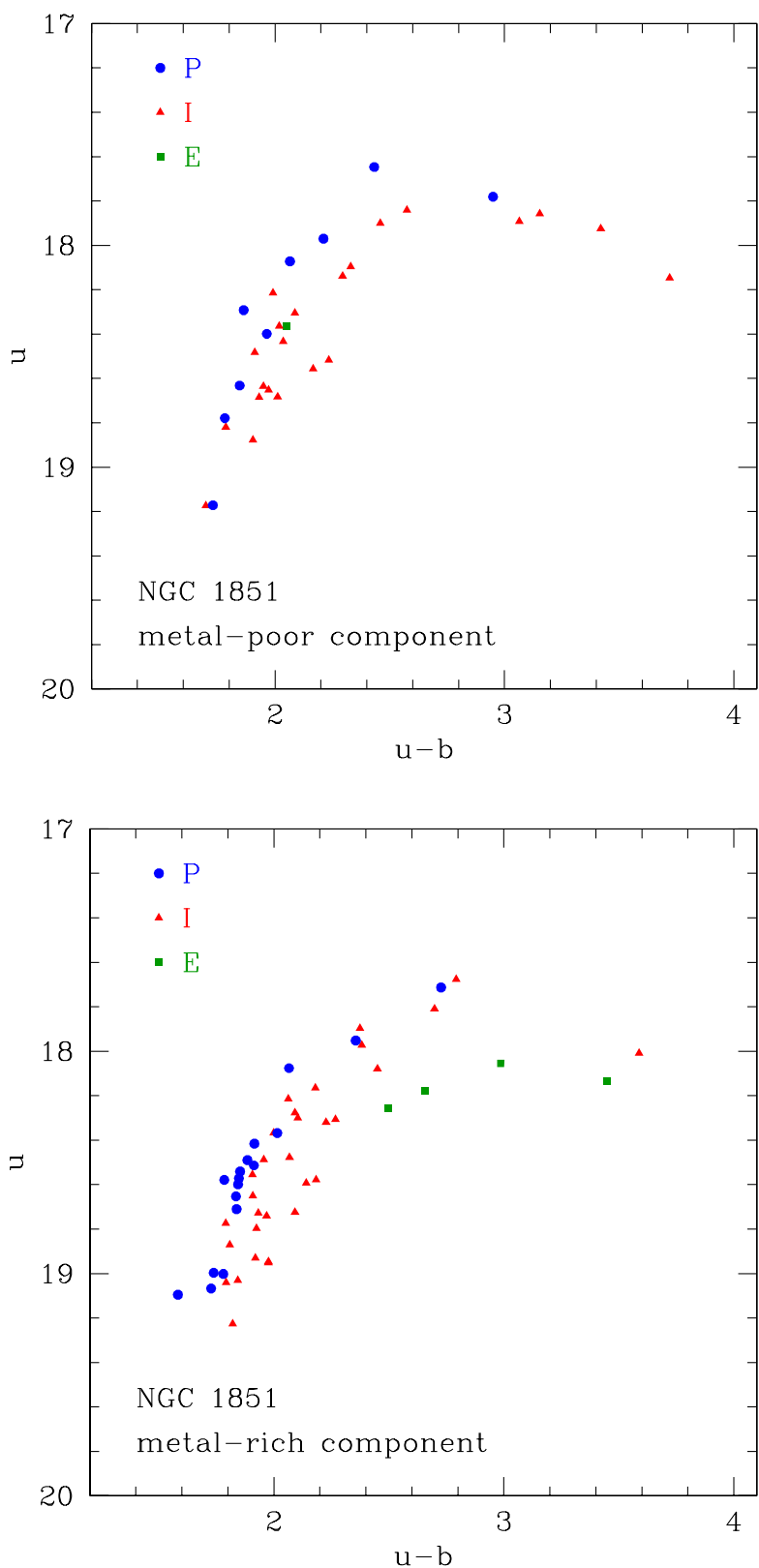

Fig. 11. Strömgren $u, u-b$ CMD for stars of the MP (upper panel) and the MR component (lower panel) in NGC 1851. In each panel, blue filled circles represent the $\mathrm{P}$ (first generation) group, the red triangles the intermediate I group, and the green squares the extreme E group of second generation stars.

To put the comparison on a more quantitative ground, we list in Table 12 the average ratios measured for the MP and MR components separately, using both the $[\mathrm{el} / \mathrm{Fe}]$ and $[\mathrm{el} / \mathrm{H}]$ abundance ratios. The value of the variable $t$ for the t-test is also listed in the table and it is used to assess whether the two averages differ statistically from each other. We found that while the $[\mathrm{el} / \mathrm{Fe}]$ mean values cannot be statistically distinguished, a significant difference is found with a high level of confidence when we compare the average $[\mathrm{el} / \mathrm{H}]$ ratios of the two components.

Thus, the metal-rich component in NGC 1851 also shows a higher level of $\alpha$-elements typically produced in corecollapse SNe.

In summary, these results are clearly telling us that the observed differences in the primordial chemical composition between the MP and MR components in NGC 1851 are due to the

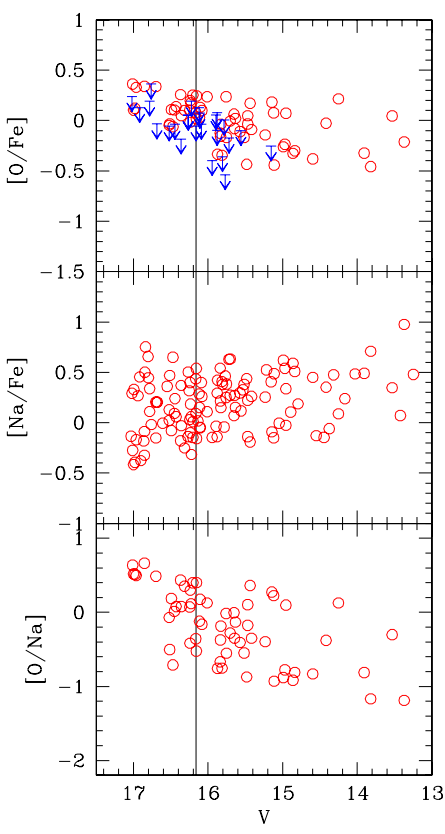

Fig. 12. $[\mathrm{O} / \mathrm{Fe}],[\mathrm{Na} / \mathrm{Fe}]$, and $[\mathrm{O} / \mathrm{Na}]$ ratios (upper, middle, and lower panel, respectively) in RGB stars of NGC 1851 as a function of $V$ magnitude. The RGB-bump luminosity level is indicated by a solid line. Upper limits in $\mathrm{O}$ are indicated by arrows.
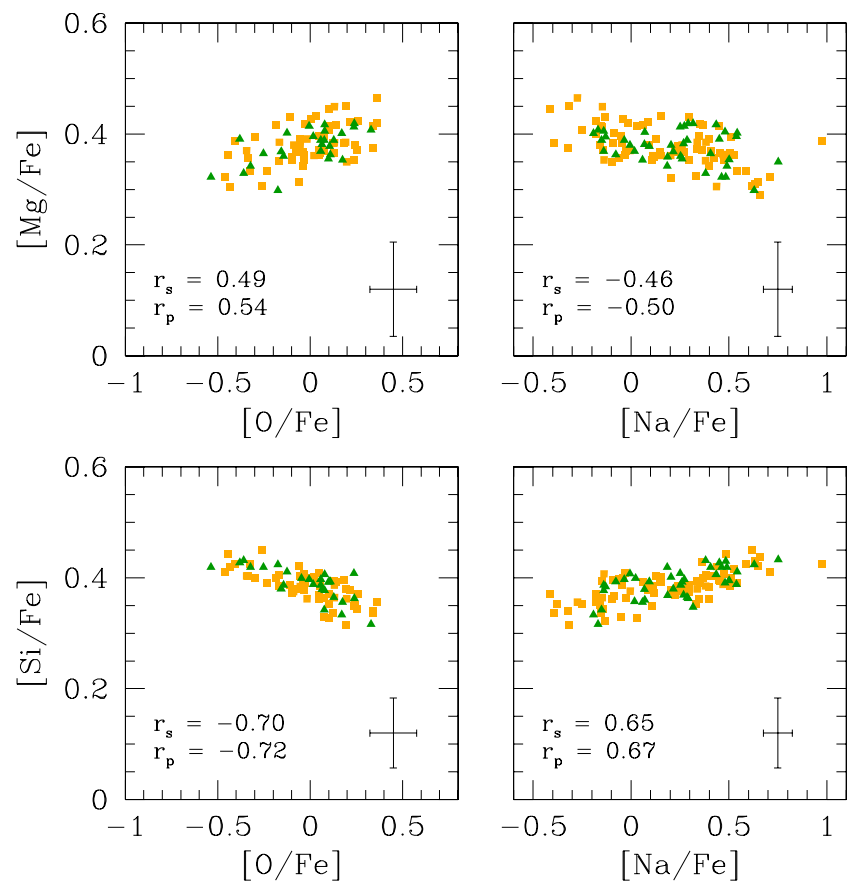

Fig. 13. Top panels: $[\mathrm{Mg} / \mathrm{Fe}]$ ratios as a function of $[\mathrm{O} / \mathrm{Fe}]$ and $[\mathrm{Na} / \mathrm{Fe}]$ for stars in NGC 1851 with GIRAFFE spectra. Bottom panels: $[\mathrm{Si} / \mathrm{Fe}]$ ratios as a function of $[\mathrm{O} / \mathrm{Fe}]$ and $[\mathrm{Na} / \mathrm{Fe}]$. In each panel, the internal error bars and the Spearman rank and the Pearson linear regression coefficients are indicated. Stars are color coded according to their metallicity: green triangles for MP stars and orange squares for MR stars.

enrichment of core-collapse $\mathrm{SNe}$ and not to type Ia $\mathrm{SNe}$, since the $\alpha$-elements track the Fe abundance and there is no significant difference in the $[\alpha / \mathrm{Fe}]$ ratios between the two populations.

How does this finding affect the anticorrelations between $\mathrm{Mg}, \mathrm{Si}$ and $\mathrm{Na}, \mathrm{O}$ seen in the previous section? We plot again 

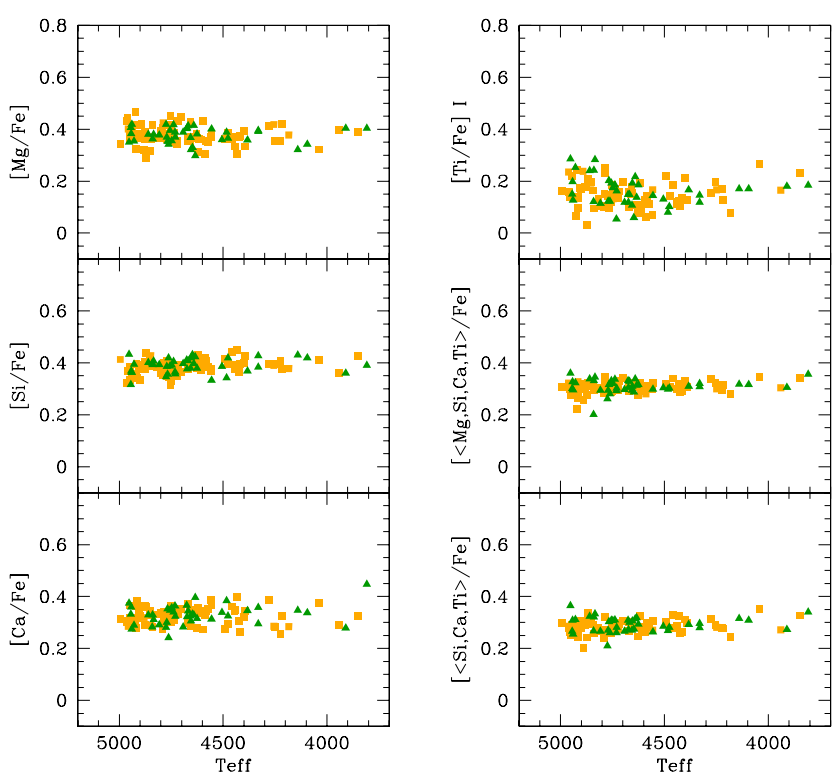

Fig. 14. Run of the abundance ratios of $\mathrm{Mg}, \mathrm{Si}, \mathrm{Ca}$ (left panels), Ti I (upper right panel) and two averages for the $\alpha$-elements (last right panels) for stars in NGC 1851 with GIRAFFE spectra. In each panel the color coding is as in Fig. 13.
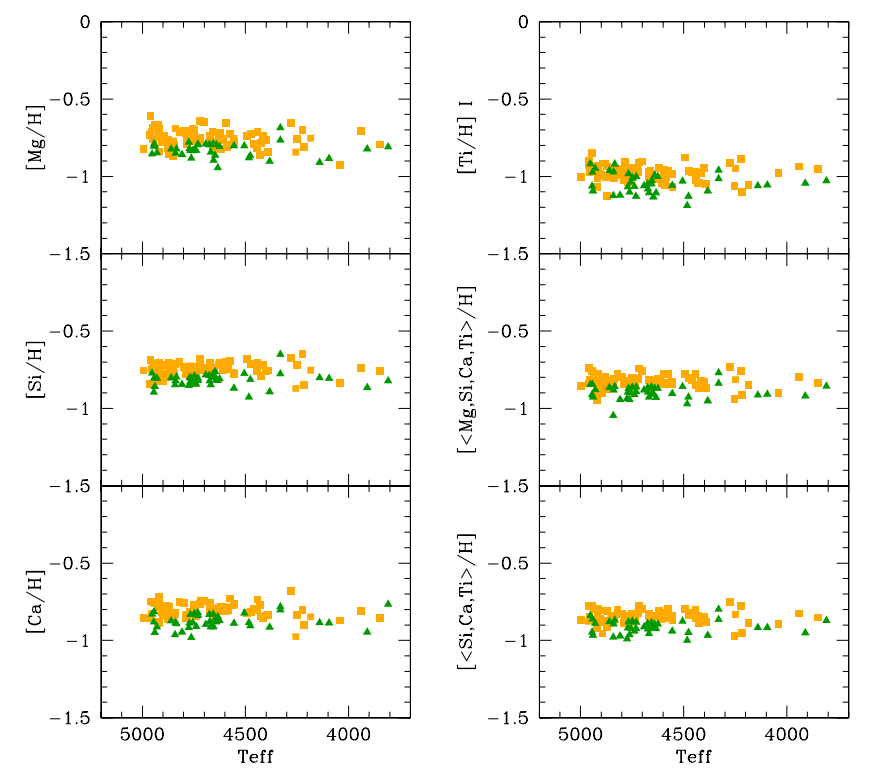

Fig. 15. As in Fig. 14 but with element ratios all with respect to $H$. The color coding is as in the previous figure.

in Fig. 16 the stars of Fig. 13, color coded as usual, but this time using the $[\mathrm{el} / \mathrm{H}]$ ratios.

From this figure, it is clear that the relations between elements involved in proton-capture reactions still hold, but for each metallicity component separately: the only difference is that the level is shifted toward higher values for the MR component. The existence of this clear anticorrelation for each metallicity group again implies that there are two originally distinct GCs. On the basis of our present knowledge, this kind of trends among light elements requires a precise chain of events and a nucleosynthesis only possible in a GC formation scenario (see Carretta et al. 2010a).

Regardless of our interpretation, we confirm that a difference in $[\mathrm{Ca} / \mathrm{H}]$ does exist in NGC 1851 . This difference was claimed
Table 12. Mean abundances from GIRAFFE spectra for stars in the MR and MP components of NGC 1851.

\begin{tabular}{|c|c|c|c|}
\hline Element & $\begin{array}{l}\text { MP component } \\
n \text { avg rms }\end{array}$ & $\begin{array}{l}\text { MR component } \\
n \text { avg rms }\end{array}$ & $t$ \\
\hline$\overline{[\mathrm{Mg} / \mathrm{Fe}]_{\mathrm{I}}}$ & $39+0.3760 .030$ & $77+0.3780 .037$ & 0.21 \\
\hline$[\mathrm{Si} / \mathrm{Fe}]_{\mathrm{I}}$ & $40+0.3890 .028$ & $77+0.3840 .029$ & 1.00 \\
\hline$[\mathrm{Ca} / \mathrm{Fe}] \mathrm{I}$ & $40+0.3290 .038$ & $78+0.3260 .033$ & 0.48 \\
\hline$[\mathrm{Ti} / \mathrm{Fe}]_{\mathrm{I}}$ & $39+0.1600 .055$ & $77+0.1510 .048$ & 0.94 \\
\hline$[\mathrm{Sc} / \mathrm{Fe}]_{\mathrm{I}}$ & $40+0.0250 .059$ & $78+0.0290 .057$ & 0.38 \\
\hline$[\mathrm{V} / \mathrm{Fe}] \mathrm{I}$ & $38-0.1250 .131$ & $75-0.1270 .109$ & 0.08 \\
\hline$[\mathrm{Cr} / \mathrm{Fe}]_{\mathrm{I}}$ & $38+0.0230 .110$ & $72+0.0640 .114$ & 1.83 \\
\hline$[\mathrm{Co} / \mathrm{Fe}] \mathrm{I}$ & $15-0.0270 .073$ & $32-0.0200 .071$ & 0.28 \\
\hline$[\mathrm{Ni} / \mathrm{Fe}]_{\mathrm{I}}$ & $40+0.0160 .078$ & $77+0.0190 .065$ & 0.24 \\
\hline$[\mathrm{Ba} / \mathrm{Fe}]_{\mathrm{II}}$ & $32+0.3950 .238$ & $69+0.5380 .203$ & 2.94 \\
\hline$[\mathrm{Mg} / \mathrm{H}]_{\mathrm{I}}$ & $39-0.8280 .047$ & $77-0.7540 .057$ & 7.39 \\
\hline$[\mathrm{Si} / \mathrm{H}] \mathrm{I}$ & $40-0.8160 .045$ & $77-0.7480 .045$ & 7.74 \\
\hline$[\mathrm{Ca} / \mathrm{H}]_{\mathrm{I}}$ & $40-0.8760 .049$ & $78-0.8070 .047$ & 7.41 \\
\hline$[\mathrm{Ti} / \mathrm{H}]_{\mathrm{I}}$ & $39-1.0450 .063$ & $77-0.9820 .053$ & 5.36 \\
\hline$[\mathrm{Sc} / \mathrm{H}] \mathrm{I}$ & $40-1.1810 .078$ & $78-1.1040 .076$ & 5.14 \\
\hline$[\mathrm{V} / \mathrm{H}]_{\mathrm{I}}$ & $38-1.3300 .132$ & $75-1.2560 .107$ & 2.96 \\
\hline$[\mathrm{Cr} / \mathrm{H}]_{\mathrm{I}}$ & $38-1.1830 .113$ & $72-1.0690 .129$ & 4.79 \\
\hline$[\mathrm{Co} / \mathrm{H}]_{\mathrm{I}}$ & $15-1.2310 .085$ & $32-1.1490 .076$ & 3.21 \\
\hline$[\mathrm{Ni} / \mathrm{H}]_{\mathrm{I}}$ & $40-1.1900 .094$ & $77-1.1130 .075$ & 4.46 \\
\hline$[\mathrm{Ba} / \mathrm{H}]_{\mathrm{II}}$ & $32-0.8090 .241$ & $69-0.5990 .206$ & 4.25 \\
\hline
\end{tabular}
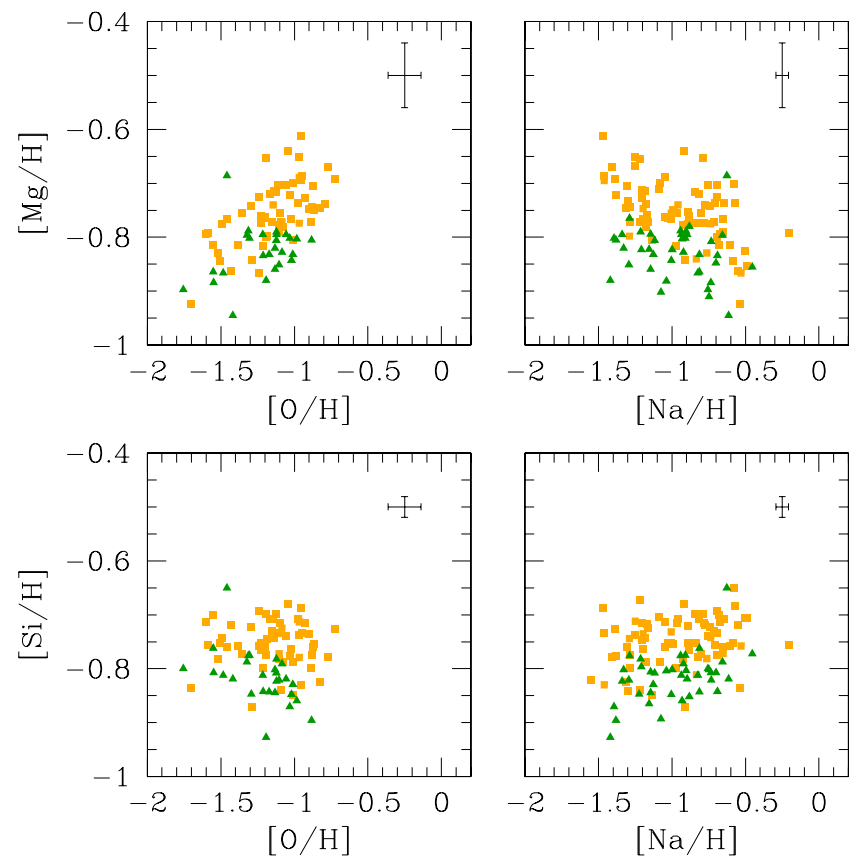

Fig. 16. Top panels: $[\mathrm{Mg} / \mathrm{H}]$ ratios as a function of $[\mathrm{O} / \mathrm{H}]$ and $[\mathrm{Na} / \mathrm{H}]$ for stars in NGC 1851 with GIRAFFE spectra. Bottom panels: [Si/H] ratios as a function of $[\mathrm{O} / \mathrm{H}]$ and $[\mathrm{Na} / \mathrm{H}]$. In each panel, the internal error bars are indicated. Stars are color coded according to their metallicity as in previous figures.

by Lee et al. (2009b) from the observed spread in their $h k$ index. A more detailed comparison between spectroscopic and photometric results will be discussed below; however, as anticipated in Carretta et al. (2010c, their Fig. 4) using Strömgren photometry, stars along the RGB in NGC 1851 are not segregated in metallicity $[\mathrm{Fe} / \mathrm{H}]$, or $[\mathrm{Ca} / \mathrm{H}]$, but only according to their belonging to either the first or second stellar generation. There is little doubt that a spread of $\mathrm{Ca}$ is present in NGC 1851, but we found that its mean level only correlates with $[\mathrm{Fe} / \mathrm{H}]$ and not $[\mathrm{Na} / \mathrm{O}]$. 

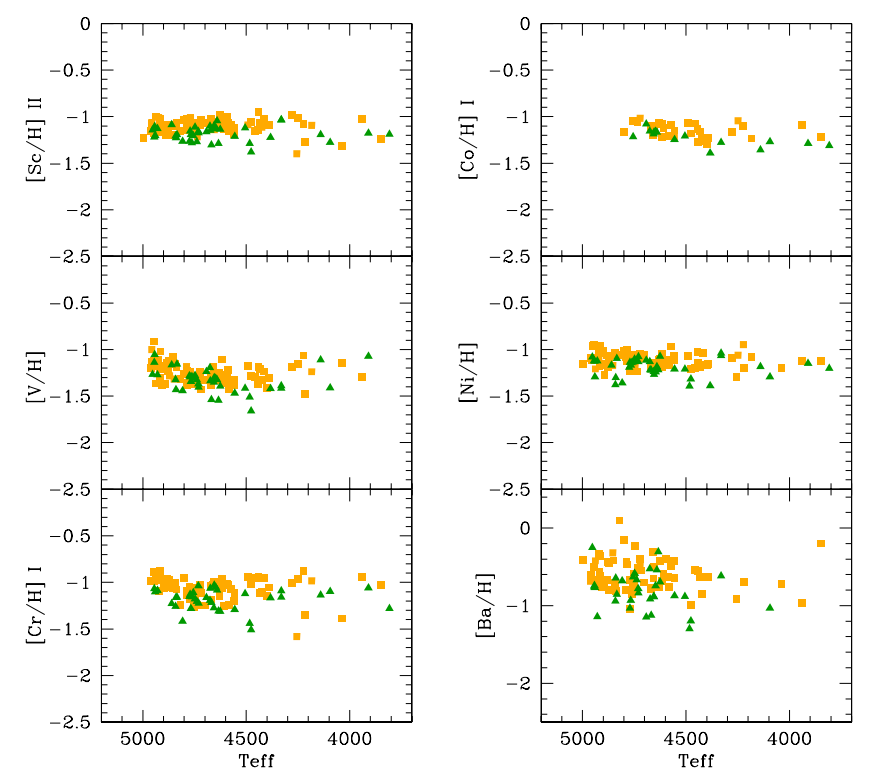

Fig. 17. Run of the abundance ratios of Fe-group elements Sc II, V, Cr, $\mathrm{Co}$, and $\mathrm{Ni}$ and the neutron-capture element $\mathrm{Ba}$ II in stars of NGC 1851 with GIRAFFE spectra. All abundance ratios are plotted with respect to $\mathrm{H}$. The color coding used in previous figures is adopted.

Our simple interpretation (the merger of two distinct clusters formed within a large parent system) offers the advantage of explaining the observation in this peculiar cluster, and at the same time accounting for other $\mathrm{GCs}$ where a spread of $[\mathrm{Ca} / \mathrm{H}]$ values is excluded by high resolution spectroscopy (such as M 4, NGC 6752, and other GCs with no metallicity spreads, see Carretta et al. 2010d).

The elements heavier than Ti, in particular the Fe-group elements (listed in Table 6), simply track the run of $[\mathrm{Fe} / \mathrm{H}]$ in NGC 1851, hence are slightly enhanced in the metal-rich component (Fig. 17). The difference between average values for the MR and MP components are again statistically significant when the $[\mathrm{el} / \mathrm{H}]$ ratios are used (Table 12 ).

\subsection{Nucleosynthesis from less massive stars: neutron-capture elements}

\subsection{1. s- and r-process contributions}

Neutron-capture elements can be produced by both a slow $s$-process, and a rapid $r$-process, where slow and rapid describe the rate of the $n$-capture with respect to the $\beta$-decay. The $r$-process is active in (some?) core-collapse SNe, while the $s$-process is mainly active in the thermally pulsing phase of intermediate-low mass stars (main component), although the light elements might also be produced in massive stars (weak component). Hence, they have very different timescales. Both $r$ and $s$-processes likely help to make the $n$-capture elements observed in NGC 1851. To estimate their relative importance, we compared the abundances of elements that in the Sun are mainly produced by the $s$ - and $r$-process. In practice (see also Carretta et al. 2010c), we considered the abundances of Ba and La as representative of $s$-process elements, and Eu to represent the contribution of the $r$-process. In the solar system, the $r$-fraction of Eu is estimated to be more than $97 \%$, while it is $<40 \%$ for the other elements (for instance, it is $\sim 18 \%$ for Ba, see e.g. Simmerer et al. 2004).

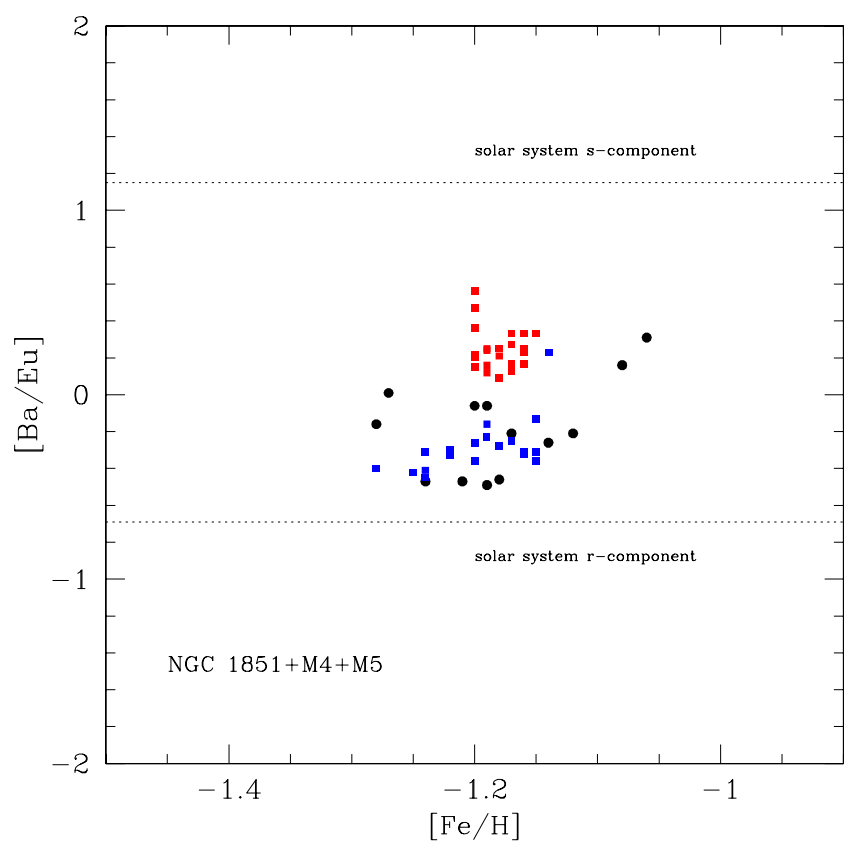

Fig. 18. Ratios $[s / r]$ in stars of NGC 1851 (black filled circles), M 4 (red squares, from Ivans et al. 1999), and M 5 (Ivans et al. 2001, blue squares). $\mathrm{Ba}$ and $\mathrm{Eu}$ are assumed to be representative of the level of $s$ and $r$-process elements, respectively.

The run of the $[\mathrm{Ba} / \mathrm{Eu}]$ ratio with $[\mathrm{Fe} / \mathrm{H}]$ in NGC 1851 is shown in Fig. 18. As a comparison, we also plotted the values available for two other GCs, which are well known to have a large difference in their abundances of neutron-capture elements (notably, those that in the Sun are mainly produced by the $s$-process): M 4 (Ivans et al. 1999) and M 5 (Ivans et al. 2001). The range in $[\mathrm{Ba} / \mathrm{Eu}]$ spanned by stars in NGC 1851 is large: about 0.80 dex, with a 0.25 dex rms scatter. There are stars with $[\mathrm{Ba} / \mathrm{Eu}]$ as high as that of $\mathrm{M} 4$, whereas others have a ratio as low as that of M 5 .

In the metal-poor stars of NGC 1851, the $s / r$ ratio is lower than solar by about 0.2 dex. It may be reproduced by assuming that about one-third of $\mathrm{Ba}$ is made by the $r$-process, and the rest by the $s$-process. An even larger fraction of other elements that in the Sun are mainly due to the s-process (e.g. Y and $\mathrm{Zr}$ ) is likely due to the $r$-process in the MP component of NGC 1851. Over the metallicity range of NGC 1851, however, there seems to be a trend of increasing $\mathrm{Ba}, \mathrm{La}$, and $\mathrm{Ce}$ with respect to $\mathrm{Eu}$ as metallicity increases. For the MR component, $[\mathrm{Ba} / \mathrm{Eu}]$ is roughly solar. This is consistent with about four-fifths of the $\mathrm{Ba}$ of the MR component being produced by the $s$-process. In this case, the $s$-process dominates also for other elements, including $\mathrm{Y}$ and $\mathrm{Zr}$.

\subsubsection{Light and heavy s-elements}

Abundances of heavy elements might also enable the heavy (second peak) to light (first peak) $s$-process elements ratios to be calculated for the stars in the sample. This ratio is important, since it can provide insight into the timescales of and the mechanisms responsible for any enrichment. In general, while it must be kept in mind that the neutron density at the site of the $s$-process does in fact control it, for a given neutron density (and metallicity) the heavy-to-light element ratio depends mostly on the stellar mass, with lower mass stars $\left(\sim 1.5-2 M_{\odot}\right)$ undergoing a larger number of thermal pulses than $\sim 3 M_{\odot}$ stars (Travaglio et al. 2004). 
The main neutron source for the mass range under consideration $\left(1.5-3 M_{\odot}\right)$ is indeed ${ }^{13} \mathrm{C}(\alpha, n){ }^{16} \mathrm{O}$, whose sensitivity to temperature is rather moderate, making the neutron-flux very similar over the mass range. A higher heavy-to-light ratio should then be related to a longer timescale (even though the overall metallicity must also be taken into consideration).

To obtain a meaningful evaluation of the heavy-to-light $s$-process ratio, the $r$-process contribution must be subtracted from the abundances of the heavy elements. To this aim, we used the following approach. We started by considering the solar $s$ - and $r$-fractions listed in Simmerer et al. (2004) for Y, $\mathrm{Zr}, \mathrm{Ba}, \mathrm{La}$, and Eu. We then assumed that the abundance ratios among different elements with similar atomic weight produced by the $r$ - and $s$-process are identical to the $r$-and $s$-components measured in the Sun for all our sample stars; for instance, we have $\log N_{\mathrm{Eu}_{s}} / \log N_{\mathrm{Ba}_{s}}=\left[\log N_{\mathrm{Eu}_{s}} / \log N_{\mathrm{Ba}_{s}}\right]_{\odot}$. This is a reasonable assumption, since these elements are close to each other in the nucleosynthesis chain. On this basis, we derived the $s$ and $r$-contribution for Eu and $\mathrm{Ba}$ for all stars. We then assumed that the $r$-process contribution for all elements scales proportionally (that is, we assumed a universal solar scaled $r$-process). This allowed us to determine the $r$-contribution for all elements in all stars. We subtracted this contribution to the abundances of all $n$-capture elements: the residual represents our best guess of the $s$-process contribution for each element in each star. This method provides reliable results insofar the $s$-contribution dominates over the $r$-one. Otherwise, our results are quite unstable, depending heavily on observational errors.

To reduce this concern, we took the average of $\mathrm{Y}_{s}$ and $\mathrm{Zr}_{s}$ to represent the light $s$-process elements and $\mathrm{Ba}_{s}$ and $\mathrm{La}_{s}$ for the heavy ones. We note that for a few stars the estimated $r$-component for $\mathrm{Y}$ and $\mathrm{La}$ accounted for the entire measured abundances of these elements (stars involved are \# 14080 and 43466 for both Y and La, and \# 39801 only for Y). In these cases, we used only $\mathrm{Ba}_{s}$ for heavy- $s$ and $\mathrm{Zr}_{s}$ for light- $s$. The remaining elements were not considered because either we conservatively deemed our abundances not accurate enough $(\mathrm{Ce})$ or the large $r$-contribution means that the results are unreliable. Plots of the behaviour of the ratio of heavy-to-light $s$-process elements as a function of $[\mathrm{Fe} / \mathrm{H}]$, measured $\mathrm{Ba}$ and $\mathrm{Eu}$ abundances $([\mathrm{Ba} / \mathrm{Fe}]$ and $[\mathrm{Eu} / \mathrm{Fe}])$, and $[\mathrm{Ba} / \mathrm{Fe}]_{s}$ are shown in Fig. 19.

Squares and triangles represent the metal-rich and the metalpoor population, respectively. The large scatter among the metalpoor population is expected, since the $r$-contribution is typically large. In the metal-rich stars, for which our results are more reliable, the $[\mathrm{hs} / \mathrm{ls}]$ ratios are typically quite high ${ }^{9}$. This suggests that there is a contribution from low-mass AGB stars $\left(M \sim 1.5-3 M_{\odot}\right)$, at least for what concerns the high-metallicity population. However, it is unlikely that stars at the very low end of this mass range $\left(M<2 M_{\odot}\right)$ have played too large a role in the chemical evolution of NGC 1851. These small mass stars do not deplete $\mathrm{O}$; a large contribution from these stars would have diluted and even erased the $\mathrm{Na}-\mathrm{O}$ anti-correlation. To maintain the Na-O anticorrelation, the contribution of the AGB stars that do not undergo HBB must be small with respect to those that do undergo this. As an exercise, we calculated the amount of mass ejected by small mass AGB (not experiencing HBB), and by more massive ones (undergoing HBB), assuming a Salpeter IMF. We found that when adopting a threshold for HBB of $M=3 M_{\odot}$,

\footnotetext{
9 We also find low and apparently constant $\mathrm{Cu}$ abundances in NGC 1851. This is consistent with a negligible contribution by the weak component of the $s$-process, since this element is thought to be overproduced by this mechanism (Sneden et al. 1991).
}
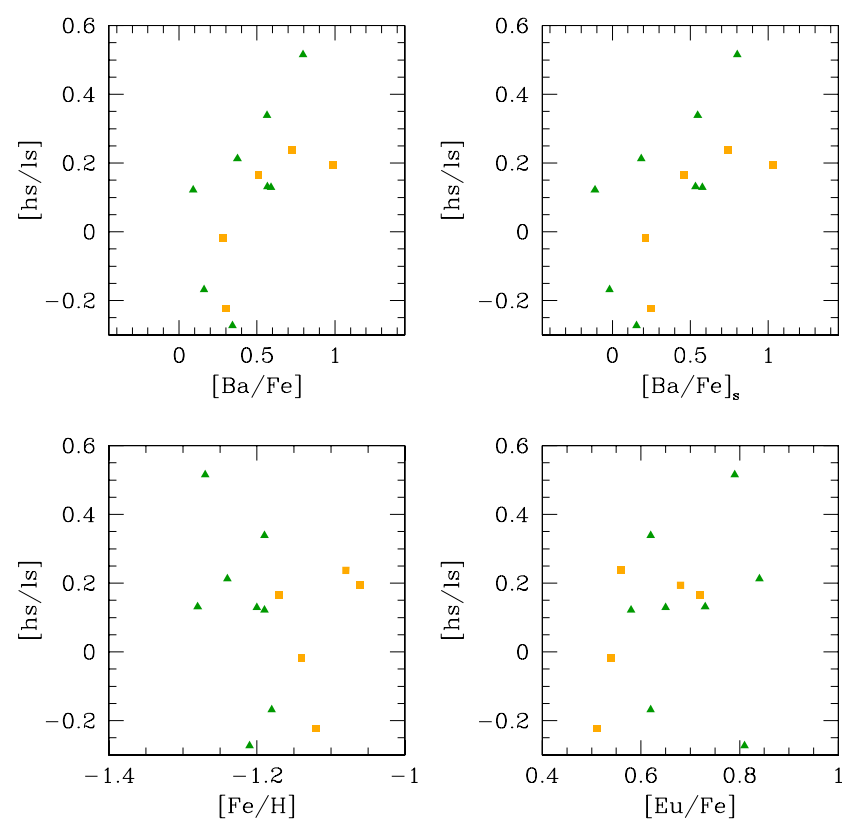

Fig. 19. $[\mathrm{hs} / \mathrm{ls}]$ as function of $[\mathrm{Fe} / \mathrm{H}]$, total $\mathrm{Ba}$ and $\mathrm{Eu}$ abundances and $\mathrm{Ba} s$-component abundance $[\mathrm{Ba} / \mathrm{Fe}]_{s}$. Squares and triangles refer to the MR and MP components, respectively.

only stars with $M>2.2 M_{\odot}$ could contribute to the second generation of NGC 1851, or else the Na-O anticorrelation would be cancelled. This is of course a schematic approach: there are likely stars that have experienced both $\mathrm{HBB}$ and thermal pulses. However, the qualitative argument that the contribution by low mass stars should be small remains valid.

We note that D'Orazi et al. (2011) used a similar approach to study the pattern of heavy elements in $\omega$ Cen. They found that this requires the presence of AGB stars even more massive than those considered here to act as the site of the $s$-process observed in that cluster.

\subsubsection{Correlations between $n$-capture and $p$-capture element abundances}

In Fig. 20 we investigated the relation between neutron-capture and proton-capture elements in our sample, using the full set of abundances available for the 13 stars with UVES spectra. In Table 13 we list the Pearson's correlation coefficient, the number of the degree of freedom and the level of statistical confidence for the linear fits made to the data in three cases: (i) using all the sample; (ii) and (iii) separating the sample in MP and MR components. Since in these plots a single star (32903) might drive part of the correlations, we highlighted it in Fig. 20 with a cross.

The first conclusion from these plots and the associated fits is that elements such as Eu and Dy, whose solar system abundances are almost totally due to the $r$-process contribution, do not present any significant correlation with proton-capture elements. In addition, the run of $\mathrm{Nd}$ with proton-capture elements is essentially flat.

Yong \& Grundahl (2008) identified a possible correlation between the abundances of the elements produced by $p$-capture an $n$-capture processes. A similar result was also found by V10. On the whole, our analysis of a far more extensive sample confirms this finding; this is most clear when we use the large sample provided by Giraffe spectra. While only a single Ba line (the one 


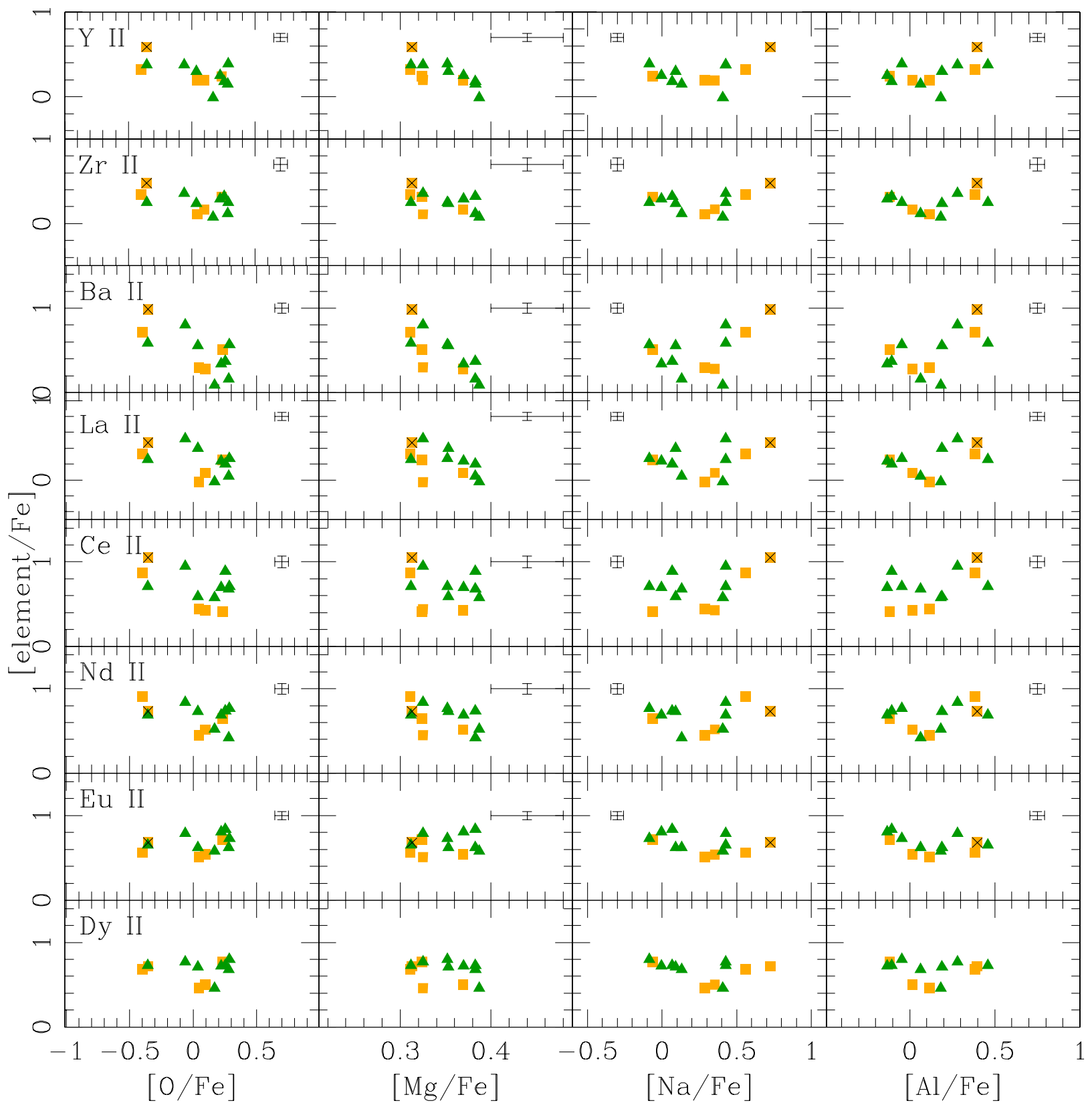

Fig. 20. Abundance ratios of neutron-capture elements in stars with UVES spectra, as a function of the proton-capture elements $\mathrm{O}, \mathrm{Mg}, \mathrm{Na}$, and $\mathrm{Al}$. Stars are separated in MR and MP components as in previous figures. The star 32903 is indicated by a cross superimposed to the symbol. Error bars indicate internal errors.

at $6141 \AA$ ) could be measured in these spectra, the correlation between $[\mathrm{Ba} / \mathrm{Fe}]$ and various indices related to the abundance of $p$-capture elements $\left([\mathrm{Na} / \mathrm{Fe}]\right.$, the Strömgren $m_{1}$ and $c_{1}$ indices, the Lee $h k$ index, etc.) is strong, at least for stars of the MR component. The level of confidence is well above $99 \%$ (see e.g., Fig. 21). While some trend with luminosity is also present, the correlations clearly hold also if we limit ourselves to the stars fainter than $V=15.5$, and still with a very high level of confidence. While all these results concern $\mathrm{Ba}$, similar correlations are also found for the other mainly $s$-elements whose lines could be measured in the UVES spectra. In Fig. 22, to enhance our "signal" we used an average of $\mathrm{Ba}, \mathrm{La}$, and $\mathrm{Ce}$ and plotted the ratio $[\langle\mathrm{Ba}, \mathrm{La}, \mathrm{Ce}\rangle / \mathrm{Eu}]$ as a function of the abundances of $p$-capture elements for stars with high resolution UVES spectra.
In this figure, we report the Spearman rank and the Pearson's correlation coefficients relative to the whole sample of 13 stars; in this case, however, almost all the relations remain statistically significant (often at a higher than $99 \%$ level of confidence) regardless of whether we separate the MP and MR components or even if the star 32903 is dropped from the sample. These correlations are not due to individual stars with anomalous values, as we see by plotting the $[\mathrm{Ba} / \mathrm{Fe}]$ ratio against the $[\mathrm{Na} / \mathrm{O}]$ abundance for the much more numerous stars with GIRAFFE spectra in Fig. 21. The correlation is again significant at a very high level of confidence for the MR stars, while the case for MP stars is more dubious.

In summary, we conclude that there is strong evidence of a correlation between $p$-capture and $n$-capture elements, at least 


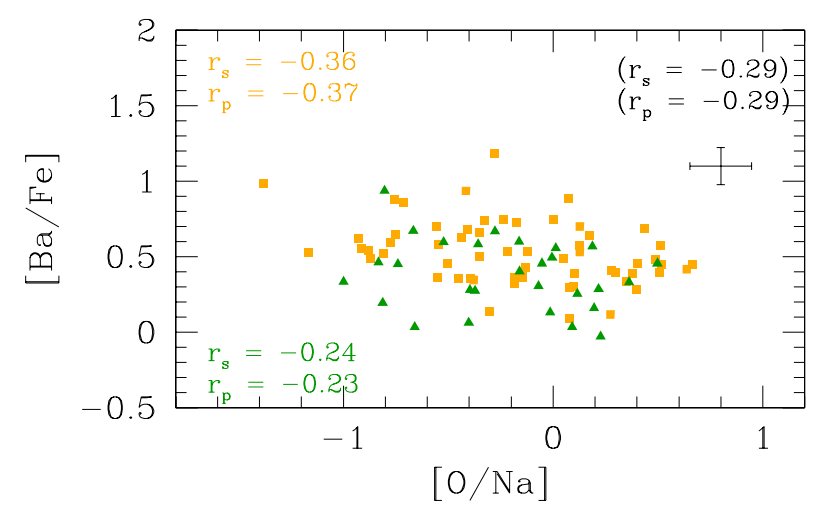

Fig. 21. $[\mathrm{Ba} / \mathrm{Fe}]$ ratios as a function of $[\mathrm{Na} / \mathrm{O}]$ for stars in NGC 1851 with GIRAFFE spectra, separated according to their sub-population. The Spearman rank coefficient $r_{\mathrm{s}}$ and the Pearson linear regression coefficient $r_{\mathrm{p}}$ are indicated for the whole sample and both sub-populations.

Table 13. Pearson's correlation coefficients, degree of freedom d.o.f., and level of significance for relations of neutron-capture vs. protoncapture elements.

\begin{tabular}{lccc}
\hline \hline Element & All & MP & MR \\
& stars & & \\
\hline Y II vs. O & $0.611195-98 \%$ & $0.476<90 \%$ & $0.733<90 \%$ \\
Y II vs. Mg & $0.711199 \%$ & $0.83699 \%$ & $0.563<90 \%$ \\
Y II vs. Na & $0.3311<90 \%$ & $0.116<90 \%$ & $0.723<90 \%$ \\
Y II vs. Al & $0.4711<90 \%$ & $0.256<90 \%$ & $0.733<90 \%$ \\
Zr II vs. O & $0.4711<90 \%$ & $0.246<90 \%$ & $0.613<90 \%$ \\
Zr II vs. Mg & $0.541190-95 \%$ & $0.516<90 \%$ & $0.593<90 \%$ \\
Zr II vs. Na & $0.2511<90 \%$ & $0.146<90 \%$ & $0.463<90 \%$ \\
Zr II vs. Al & $0.2411<90 \%$ & $0.106<90 \%$ & $0.553<90 \%$ \\
Ba II vs. O & $0.691199 \%$ & $0.566<90 \%$ & $0.783<90 \%$ \\
Ba II vs. Mg & $0.781199 \%$ & $0.86699 \%$ & $0.703<90 \%$ \\
Ba II vs. Na & $0.4411<90 \%$ & $0.126<90 \%$ & $0.643<90 \%$ \\
Ba II vs. Al & $0.551195 \%$ & $0.386<90 \%$ & $0.753<90 \%$ \\
La II vs. O & $0.481190 \%$ & $0.436<90 \%$ & $0.663<90 \%$ \\
La II vs. Mg & $0.531190-95 \%$ & $0.72695-98 \%$ & $0.563<90 \%$ \\
La II vs. Na & $0.2111<90 \%$ & $0.026<90 \%$ & $0.513<90 \%$ \\
La II vs. Al & $0.3811<90 \%$ & $0.246<90 \%$ & $0.593<90 \%$ \\
Ce II vs. O & $0.4711<90 \%$ & $0.096<90 \%$ & $0.94398-99 \%$ \\
Ce II vs. Mg & $0.2311<90 \%$ & $0.296<90 \%$ & $0.613<90 \%$ \\
Ce II vs. Na & $0.4511<90 \%$ & $0.126<90 \%$ & $0.86390-95 \%$ \\
Ce II vs. Al & $0.4511<90 \%$ & $0.076<90 \%$ & $0.93398 \%$ \\
Nd II vs. O & $0.4511<90 \%$ & $0.306<90 \%$ & $0.743<90 \%$ \\
Nd II vs. Mg & $0.4611<90 \%$ & $0.596<90 \%$ & $0.613<90 \%$ \\
Nd II vs. Na & $0.1411<90 \%$ & $0.096<90 \%$ & $0.443<90 \%$ \\
Nd II vs. Al & $0.2911<90 \%$ & $0.036<90 \%$ & $0.643<90 \%$ \\
Eu II vs. O & $0.2911<90 \%$ & $0.206<90 \%$ & $0.063<90 \%$ \\
Eu II vs. Mg & $0.1411<90 \%$ & $0.066<90 \%$ & $0.373<90 \%$ \\
Eu II vs. Na & $0.4111<90 \%$ & $0.356<90 \%$ & $0.213<90 \%$ \\
Eu II vs. Al & $0.3811<90 \%$ & $0.526<90 \%$ & $0.133<90 \%$ \\
Dy II vs. O & $0.0611<90 \%$ & $0.126<90 \%$ & $0.263<90 \%$ \\
Dy II vs. Mg & $0.2811<90 \%$ & $0.536<90 \%$ & $0.583<90 \%$ \\
Dy II vs. Na & $0.3111<90 \%$ & $0.426<90 \%$ & $0.023<90 \%$ \\
Dy II vs. Al & $0.3811<90 \%$ & $0.146<90 \%$ & $0.163<90 \%$ \\
\hline & & &
\end{tabular}

for the MR component of NGC 1851. This correlation is rarely found among GCs, hence is a peculiar property of NGC 1851.

\subsubsection{Comparison with previous studies}

Other investigators have published abundances of $n$-capture elements in NGC 1851. Yong \& Grundahl (2008) found large
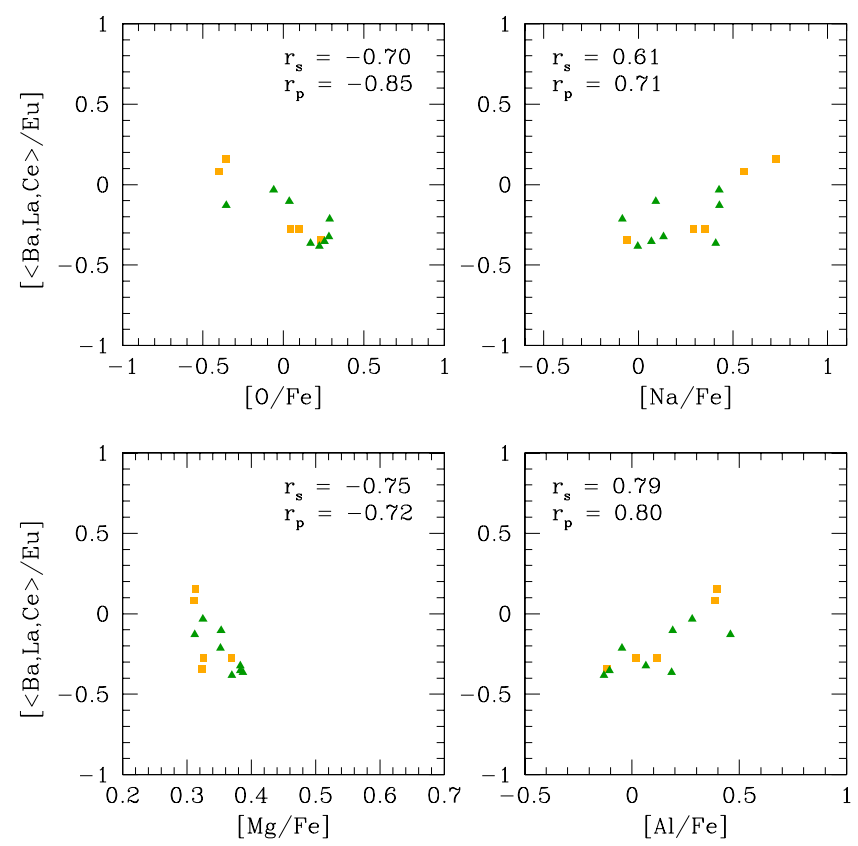

Fig. 22. The ratio $[\langle\mathrm{Ba}, \mathrm{La}, \mathrm{Ce}\rangle / \mathrm{Eu}]$ as a function of the proton-capture elements $\mathrm{O} . \mathrm{Na}, \mathrm{Mg}$, and $\mathrm{Al}$ for stars with UVES spectra of the MP (green symbols) and MR (orange symbols) component in NGC 1851. Both the Spearman rank correlation coefficient and Pearson linear regression correlation coefficient are indicated in each panel.

star-to-star variations in a sample of eight bright giants. In particular, they found that the abundances of $\mathrm{Zr}$ and $\mathrm{La}$ were anticorrelated with $\mathrm{O}$ and correlated with $\mathrm{Al}$. While this result closely agrees with ours, we found that only their correlations involving $\mathrm{La}$ are actually statistically significant (here we assume that a level of confidence below $90 \%$ for the Pearson's correlation coefficient implies that the associated correlation is not significant).

Five stars among our UVES sample (25859, 29719, 32903, 39801 , and 41689) are in common with V10, who report abundances of $\mathrm{Fe}, \mathrm{Na}$, and $\mathrm{O}$ generally in reasonable agreement with ours. However, our $\mathrm{Y}$ and $\mathrm{Ba}$ abundances are systematically higher than V10, in some cases by as much as 0.3 and 0.6 dex, respectively. Given that V10 listed neither the adopted atmospheric parameters nor explicitly the atomic parameter for these particular elements, we cannot attempt to trace the source of this discrepancy. We only note that the abundances derived from the four $\mathrm{Y}$ and $\mathrm{Ba}$ lines are in good agreement with each other in our data.

\section{Comparison of spectroscopy and photometry in NGC 1851}

NGC 1851 is clearly a complex GC. Using our unprecedentedly large dataset of chemical abundances we may aim to perform a better tagging of the observed broadening and/or splitting of the photometric sequences and to achieve a deeper insight into their origin.

In Fig. 23, we summarize in six CMDs the results of a first screening of RGB stars in NGC 1851 using the $y$ Strömgren magnitude and the $h k$ index kindly provided by 


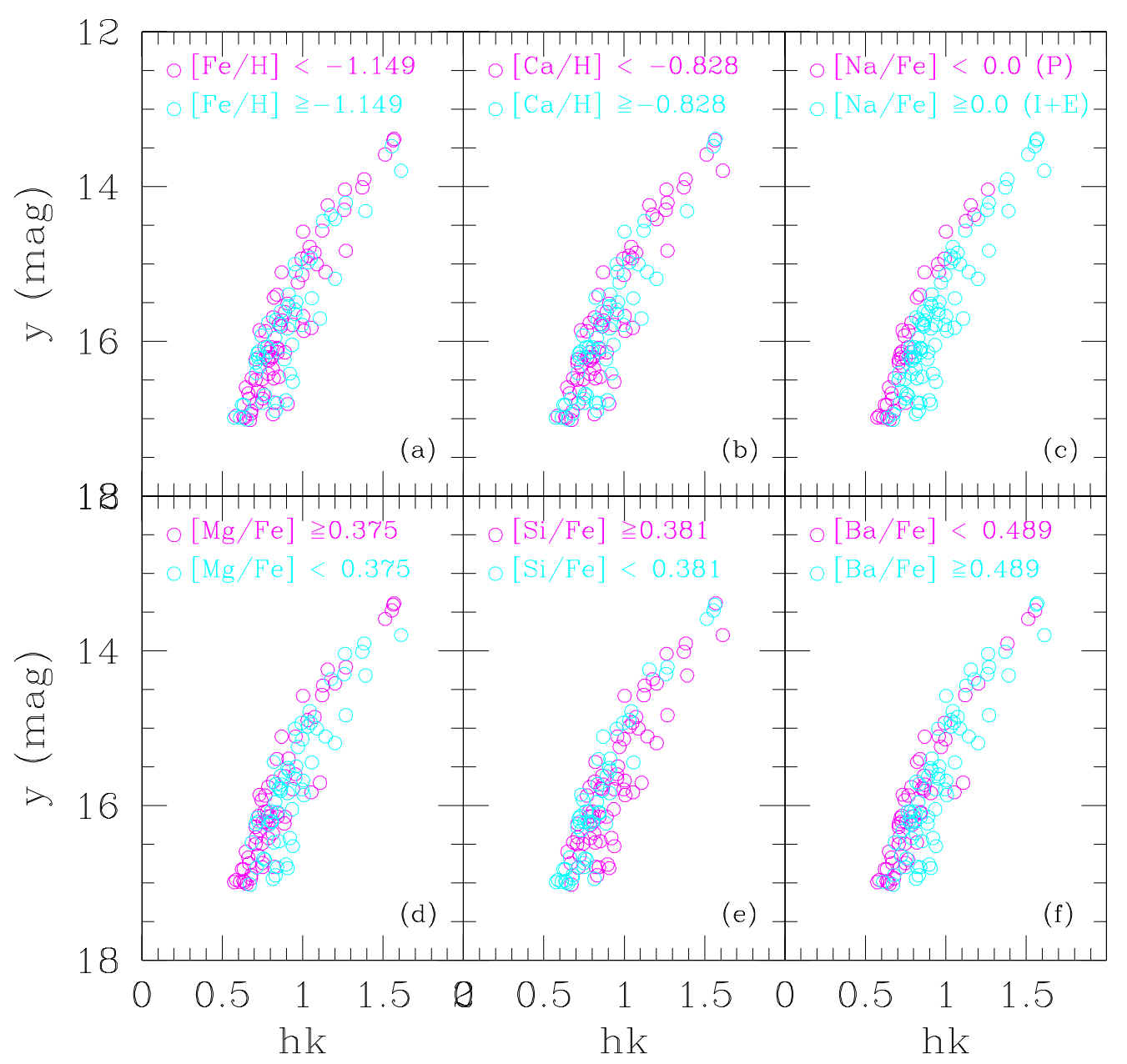

Fig. 23. RGB stars in common between our analysis and that of Lee et al. (2009a) in the $y-h k$ plane from Strömgren photometry. In each panel, stars are color coded according to their abundances derived in the present study. The elements and the separating values are indicated in each panel.

Prof. Jae-Woo Lee ${ }^{10}$. In each panel, we plot the giants of NGC 1851 in common between the present study and the work of Lee and collaborators (121 stars), colour-coded according to their abundances, higher or lower than the average value for the common sample, listed in each panel of Fig. 23.

Before discussing these plots, we note that within NGC 1851, $h k$ is extremely well correlated with the Strömgren index $m_{1}$ (see Fig. 24). However, from our result, these indices do neither appear to trace the metallicity of NGC 1851 stars (panel (a)), nor seem to be well correlated with a difference in $[\mathrm{Ca} / \mathrm{H}]$, as shown in panel (b). Admittedly, as discussed in Sect. 4.2, the difference in $[\mathrm{Fe} / \mathrm{H}]$ between the MR and MP components in NGC 1851 is small and might not be the most effective way of separating stellar components in this peculiar cluster. However, the separation between these components is real, as shown by their different radial distributions (Fig. 1 in Carretta et al. 2010c). Nevertheless, the $h k$ index seems to be insensitive to this difference.

On the other hand, in monometallic clusters $m_{1}$ measures the strength of the near-UV CN bands, and is strongly correlated

\footnotetext{
10 We verified that the Lee photometry agrees within about 0.05 mag with the Strömgren photometry by Grundahl, but in this case we adopt the first one since it is fully consistent with the $h k$ index $(h k=(C a-b)-(b-y))$.
}

with $\mathrm{N}$ abundances in metal-rich clusters (see Carretta et al. 2011). It may well be used to separate first and second generation stars in NGC 1851 (see Fig. 25). Hence, it is unsurprising that $h k$ also seems to be quite efficient in separating first and second generation stars in NGC 1851, judging from panel (c) in Fig. 23. In this panel, we did not adopt the average value for $[\mathrm{Na} / \mathrm{Fe}](+0.189$ dex for stars in common between ourselves and Lee et al.), but we chose the value 0.0 that in NGC 1851 divides first and second generation stars according to the definition of Carretta et al. (2009a). The two stellar generations can be clearly distinguished in the $y-h k$ plane. The same result also holds using the ratio $[\mathrm{O} / \mathrm{Na}]$, although the separation is not so clean as in panel (c) because of upper limits and/or larger errors in the $\mathrm{O}$ abundances.

The efficiency of $h k$ in separating first and second generation stars is also supported by the CMDs in panels (d) and (e). The separation of first and second generation stars using $\mathrm{Mg}$ and $\mathrm{Si}$ is of course less accurate, because the spread in abundances is not very large. However, the most noticeable result in these two panels is that the two groups are specularly selected, as expected because of the anticorrelation of $\mathrm{Mg}$ and $\mathrm{Si}$.

The final panel (f) shows how stars are segregated along the split RGB in NGC 1851 according to their Ba abundances. In this plane (but also in the Strömgren $v, v-y$ CMD, where the split is even clearer), only Ba-rich stars are found on the reddest 


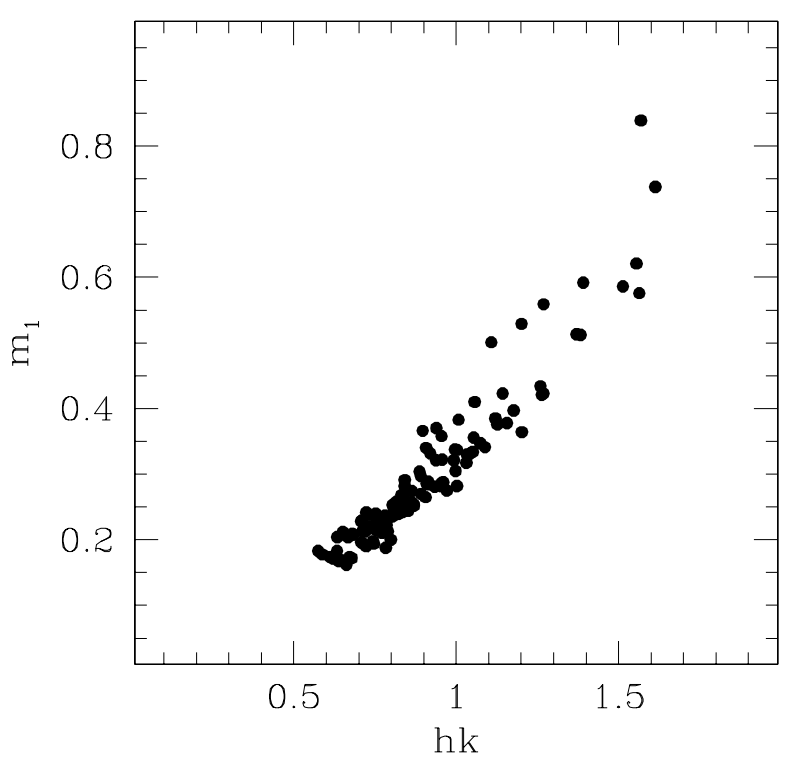

Fig. 24. Plot of $m_{1}$ and $h k$ for stars in NGC 1851 in common between our work and Lee et al. (2009a). Stars with higher values for $m_{1}$ and $h k$ are the brighter members of our sample.

section of the RGB, whereas Ba-poor stars are mostly (but not exclusively) restricted to the bluest side of the RGB. This finding confirms and strengthens a similar result by V10, based on a much more limited sample. At a given level of magnitude, all stars with high values of the $h k$ index are more Ba-strong than the average $[\mathrm{Ba} / \mathrm{Fe}]$ value, although there is not a one-to-one correspondence between $\mathrm{Ba}$ and $h k$.

To summarize the results of this section, we have found that in NGC 1851 the $h k$ index seems to be related to the dichotomy first/second generation, but not to the $\mathrm{Ca}$ abundance: in Fig. 26, we also show the direct correspondence between $h k$ and our $[\mathrm{Ca} / \mathrm{H}]$ values from spectroscopy, and we do not see any correlation.

In Carretta et al. (2009b), we demonstrated that in CMDs including the $u$ band, second generation stars that are N-enhanced, as expected from the full action of the complete CNO-cycle, are spread out to the red, along the RGB. The link between chemical composition and observed colours in first and second generation stars is examined in detail in another paper (Carretta et al. 2011), but from both Figs. 11 and 23 we can conclude tentatively that $h k$ separates stars that are N-rich and N-poor, respectively. This result is unexpected, given the definition of the Ca narrow-band index of Lee et al. (2009).

\subsection{The effect of (neglecting) carbon}

Judging from panels (c) and (f) of Fig. 23, there is a global correspondence between $\mathrm{Ba}$-rich and $\mathrm{N}$-rich stars, but there are exceptions. This is a significant difference with respect to the very tight sequences shown by V10 with their limited sample, and it suggests that this is not the whole story.

Another possible player to be considered might be the carbon abundance. In another paper (Carretta et al. 2011), we studied in detail the effect of variations in both the $\mathrm{N}$ and $\mathrm{C}$ abundances on the Strömgren photometry. Here we only summarize in Fig. 28 the main results relative to the $\mathrm{C}$ abundance variations. Briefly, we used a model atmosphere with the parameters $T_{\text {eff }}=4478 \mathrm{~K}$, $\log g=1.4$, and $[\mathrm{A} / \mathrm{H}]=-1.23$ and computed two synthetic

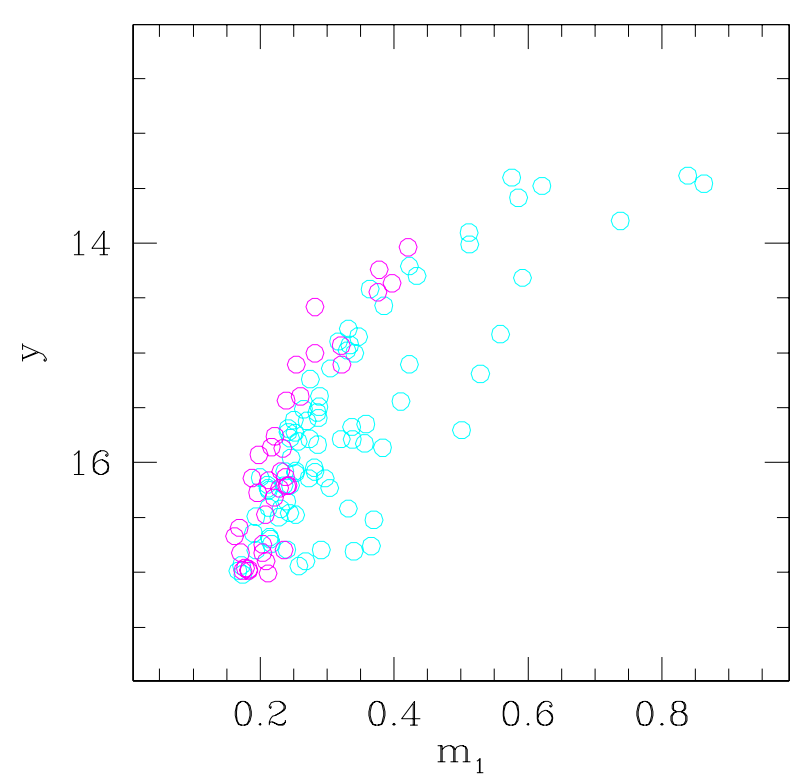

Fig. 25. Plot of $m_{1}$ vs. $y$ for stars in NGC 1851, separated into first (P, in magenta) and second (IE, in light blue) generation stars.

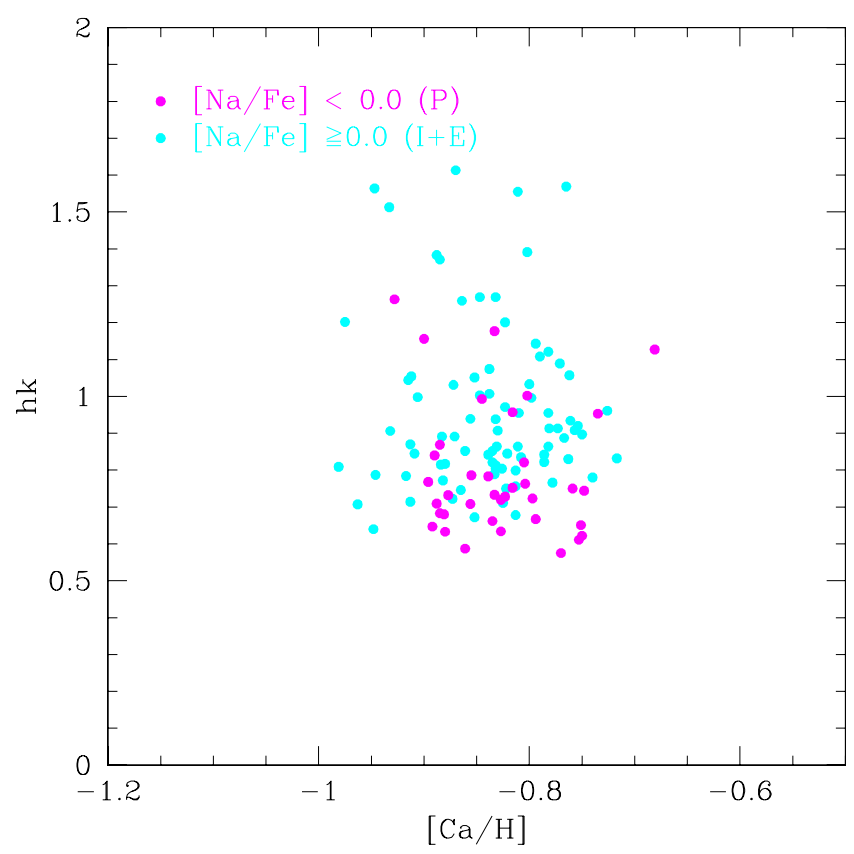

Fig. 26. $h k$ index by Lee and collaborators as a function of the $[\mathrm{Ca} / \mathrm{H}]$ ratio derived in the present study for giants in NGC 1851.

spectra for a C-rich, N-rich and a C-rich, N-poor case, respectively ${ }^{11}$. Afterwards, we evaluated the differences in magnitude with respect to the reference case of a $\mathrm{C}$-normal, $\mathrm{N}$-poor star, and we plot in the left and right panels of Fig. 28 the displacements due to variations in only $\mathrm{N}$ (red line) and in $\mathrm{C}$ only (blue line).

From this exercise, it can be seen that for $\mathrm{N}$-poor stars, the impact of varying $\mathrm{C}$ is modest. The main reason is that in these stars $\mathrm{O}>\mathrm{C}$, hence the $\mathrm{CN}$ bands are not very strong. On the other hand, for N-rich stars a change in the $\mathrm{C}$ abundance has

\footnotetext{
11 In these computations, we adopted $[\mathrm{O} / \mathrm{Fe}]=-0.1$ dex for the $\mathrm{C}$-rich, $\mathrm{N}$-rich case and $[\mathrm{O} / \mathrm{Fe}]=+0.4$ dex for the $\mathrm{C}$-rich, $\mathrm{N}$-poor case.
} 


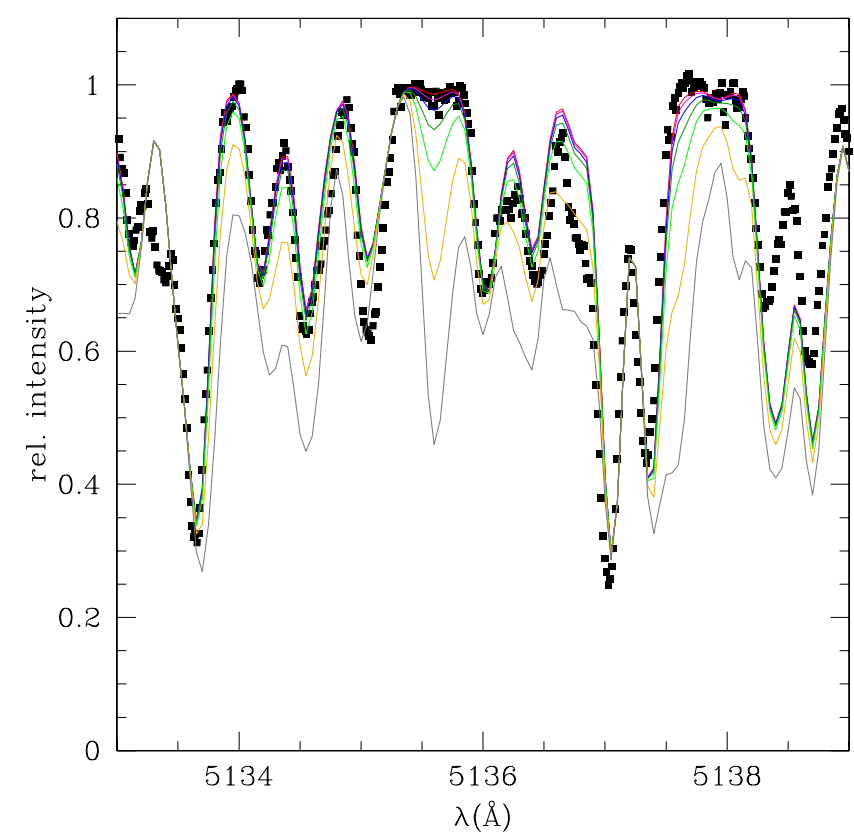

Fig. 27. Portion of the UVES spectrum of star 32903 in the $C_{2}$ region at $5135 \AA$ (filled squares) with synthetic spectra computed for various $\mathrm{C}$ abundances $([\mathrm{C} / \mathrm{Fe}]=-0.5,-0.4,-0.3,-0.2,-0.1,0.0,+0.1)$.

a notable effect, particularly in the $v$ filter. In turn, this implies large variations in indices such as $c_{1}$ and $m_{1}$.

From these computations and from the CMDs in Fig. 28, we might conclude that stars on the redder sequence of NGC 1851 are quite good candidates to be C-rich and N-rich (hence O-poor). On the other hand, the C-rich and N-poor stars (i.e. O-rich) may not be separated from the other N-poor and C-normal stars in any of the Strömgren indices. This might well explain why the reddest RGB sequence consists only of a slender strip of a few stars: they might not be all the C-rich stars present in the cluster, but only those that are also O-poor. In general, it is likely that the C-rich stars in NGC 1851 would coincide with the Ba-rich stars, and might correspond to our definition of the MR population (Sect. 4.2), related to both the brighter SGB and the red $\mathrm{HB}$ according to their number ratios.

However, the evidence supporting this view is currently limited to the Strömgren photometry. We did not find evidence of $\mathrm{C}_{2}$ in our spectra, even for the most Ba-rich (and also metal-rich) stars with UVES spectra (stars 32903 and 41689: see Fig. 27). In the first case, we can even set a quite stringent upper limit of $[\mathrm{C} / \mathrm{Fe}]<-0.4$, which is incompatible with the assumption of $\mathrm{C} \sim \mathrm{O}$ on which we base the scenario discussed in this section. It is of course possible that $\mathrm{C}$ has been transformed into $\mathrm{N}$ in these very bright giants, and that these $\mathrm{CN}$ bands are very strong, owing to a very large excess of $\mathrm{N}$. Unfortunately, we have been unable to prove this with the present spectroscopic data.

\section{Discussion and conclusions}

Several analyses of observations of NGC 1851 suggest that two (originally) distinct objects may have concurred to form the currently observed cluster: the bimodal distribution of the $\mathrm{HB}$, the double/split SGB, and the splitting recently found in the RGB. To these observations, in Carretta et al. (2010c) we added a distinct radial distribution where the MP component is more centrally concentrated. When coupled with the existence of the $\mathrm{Na}-\mathrm{O}$ anticorrelation in each component on the RGB, although with different primordial/polluted ratios of stars, this series of evidence points toward the existence of two merged clusters. The results from the previous section are in reasonable agreement with a scenario where the two clusters also differ in terms of the average level of $\mathrm{Ba}$ (and probably also $\mathrm{C}$ ) abundance, with however additional complications that we note in Sect. 6.1.

Our idea offers the advantage of accounting for a number of observations with the minimum number of assumptions. In most respects, these essentially reduce to only one, that of an age difference of about 1 Gyr between the two putative clusters (since the metallicity difference - the other necessary assumption - is actually measured from our data). The age difference stems in part because we can exclude a significant contribution from He variations: the moderate extensions of both the Na-O and $\mathrm{Al}-\mathrm{Mg}$ anticorrelations indicate that they must be irrelevant. This minor role of He variations is moreover confirmed by the constant luminosity level between the red and the blue $\mathrm{HB}$ (see Cassisi et al. 2008). An age difference of $\sim 1 \mathrm{Gyr}$ is also compatible with the higher level of $s$-process elements found for the MR component.

The combination of age and metallicity differences implies a difference of only $0.04 M_{\odot}$ in the masses of stars currently on the RGB, which is compatible with the HB but results in a negligible segregation of the two populations in the region of the cluster we observed. A primordial segregation between the MP and MR populations might then have survived the following dynamical evolution.

Finally, there might also be a difference in the $\mathrm{C}$ content between the two putative GCs; this is not only expected if the MR one is about 1 Gyr younger (allowing for the contribution of AGB stars with masses $<3 M_{\odot}$ ), but could also account for the observed Strömgren colours. However, we did not detect any $\mathrm{C}_{2}$ line in our spectra, which possibly excludes there being a significant $\mathrm{C}$ enhancement (unless this is cancelled by evolutionary effects).

In summary, most observations concerning NGC 1851 can be explained by the existence of two sub-populations. The first population contains about $35-40 \%$ of the stars, and behaves like a small GC, with normal Na-O anticorrelations and normal features. The only peculiar characteristic is maybe a number of primordial stars higher than the average $\sim 30 \%$ typical of GCs. This first population may be linked to the faint SGB (Milone et al. 2008) and to the BHB and be more centrally concentrated in NGC 1851, according to our RGB sample. A second population, formed by the remaining $60-65 \%$ of stars, corresponds to our MR component, is more rich in Ba and $s$-process elements, has, on average, stronger $h k$ indices, and Strömgren photometric indices that are consistent with an overabundance of $\mathrm{C}$. This population, that is possible to identify with the bright SGB and the RHB, is not very different in terms of either $[\mathrm{Fe} / \mathrm{H}]$ or $[\mathrm{Ca} / \mathrm{H}]$ from the first one, although it is clearly more enriched in these elements. The most significant difference, however, seems to concern the products of the third dredge-up, which implies an age difference large enough to allow low mass stars to contribute. We also note that even if the $\mathrm{C}$ abundance of the MR population is perhaps higher than that of the MP population, the $\mathrm{C}+\mathrm{N}+\mathrm{O}$ sum is probably not very different $(0.1-0.2 \mathrm{dex}$, mainly concentrated in the $\mathrm{C}$ term), because in all cases $\mathrm{C} \ll(\mathrm{N}+\mathrm{O})$. The contribution of low mass $\left(\sim 2-3 M_{\odot}\right)$ stars is also required because of the heavy-to-light ratio inferred for the $s$-process elements. 


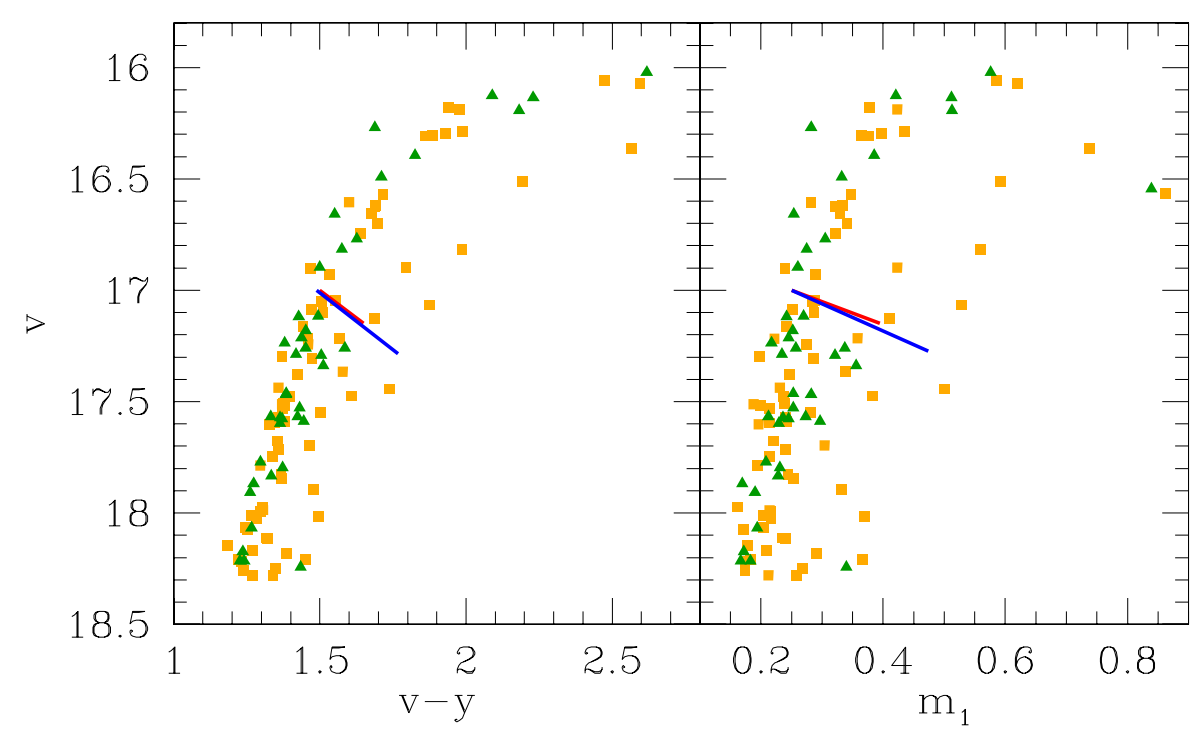

Fig. 28. Strömgren $v, v-y$ and $v, m_{1}$ for the MP and MR components in NGC 1851. In both panels, the red and blue lines indicate the effect of a $\mathrm{N}$ and a $\mathrm{C}$ variation, respectively, on the stars' colours (see text).

\subsection{The correlation between proton- and neutron-capture elements}

We confirm and extend the results of Yong \& Grundahl (2008). In NGC 1851, the abundances of $s$-process dominated elements seems to be strictly related to those of the light elements involved in $p$-capture reactions. The level of neutron-capture elements increases for stars where the signature of processing by $\mathrm{H}$-burning at high temperature is more marked. While this signature is very distinctive for the MR population, it is not obvious for the MP population, depending on the membership of a couple of stars.

This observation bears a potential problem, depending on the mechanism assumed for the origin of the $s$-process elements (e.g. Käppeler 1999). If, as also suggested by the high heavy-to-light ratio, they were forged by the main component (e.g. by low mass stars with $M_{\text {ZAMS }} \sim 1.5-3 M_{\odot}$ during the AGB phase), then the evolutionary times of these stars range from about 340 Myr up to about 2 Gyr (e.g. Schaller et al. 1992). However, if AGB stars with 4-8 $M_{\odot}$ are responsible for the $p$ capture elements, the release of polluted matter from these stars is expected as early as about $40 \mathrm{Myr}$ and as late as $160 \mathrm{Myr}$ after their birth (the timescale for the production of $p$-capture elements would be even shorter were they produced by massive stars). This timescale problem could be overcome only if the gas cumulated in a reservoir that does not form stars for several $10^{8} \mathrm{yrs}^{12}$.

Such a time delay can perhaps be accommodated in models with a cooling flow as tentatively proposed by D'Ercole et al. (2008) if e.g. a threshold in the mass of the gathering gas is necessary to trigger the formation of the second generation. In addition, whether the correlation between $n$ - and $p$-capture elements were continuous, dilution of the material of this reservoir with unprocessed material would be required. Unfortunately, our data are insufficiently precise to determine whether this last hypothesis is valid.

An alternative possibility would be that the same stars produced both $n$ - and $p$-capture elements, as suggested by D'Antona et al. (2011) to explain a similar problem for $\omega$ Cen.

\footnotetext{
12 Note that this is not the long delay of $\sim 1$ Gyr between MP and MR populations discussed at the beginning of Sect. 7 .
}

Such an hypothesis meets several difficulties, for instance the lack of correlation between $n$ - and $p$-capture elements in most GCs. Furthermore, the only known mechanism producing the $n$-capture elements in massive stars (the weak component: Raiteri et al. 1991) is a secondary mechanism (then likely negligible in metal-poor stars), which preferentially produces the less massive among the elements along the $s$-chain, and for instance a significant fraction of $\mathrm{Cu}$. At variance with the case of M 22 (Marino et al. 2011), we do not find any sign of $\mathrm{Cu}$ production within NGC 1851, and the heavy-to-light element ratio is large.

It then seems that the correlations between $p$ - and $n$-capture elements in NGC 1851 observed by us and Yong \& Grundahl (2008) place serious constraints on the scenario of pollution in GCs from AGB stars.

\subsection{Hunting for the ancestral galaxy}

The idea proposed by van den Bergh (1996) that GCs with a composite CMD are the result of a merger of two parent GCs within the past equivalent to a present-day dwarf spheroidal, offers several attractive ways of explaining the peculiarities observed in NGC 1851.

First, the $N$-body simulations of Makino et al. (1991) show that orbital angular momentum is transferred at earlier stages to the escaping particles: this process causes the shrinkage of the orbit, hence the onset of a merger. A large loss of stars should occur at early phases of formation of a proto-GC, according to the observed composition of first and second generation stars (see e.g. Carretta et al. 2010a; D'Ercole et al. 2010; Conroy 2011). Should the early cluster evolution occur in the environment of a dSph, this large mass loss might increase the probability of the merger process.

If the clusters originated in the same dwarf galaxy, they might have both spiralled to the centre (by dynamical friction) and then merged to form the currently observed object. A necessary condition for this chain of events is that the dSph must have survived for at least a few Gyr before interacting with our Galaxy. Cases of dSphs hosting a system of GCs of their own are well known (Fornax, and the disrupting Sagittarius dwarf). 
On the other hand, the different density distributions of MP and MR stars suggests that the merging of the two clusters occurred not too long in the past, less than a few Gyr ago. Since this merging should have happened before the putative dwarf spheroidal dissolved, it is possible that the remains of this ancestral, parent galaxy can still be traced around NGC 1851. A study by Olszewski et al. (2009) found that the cluster is embedded in a halo $500 \mathrm{pc}$ across, corresponding to about $0.1 \%$ of the dynamical mass of NGC 1851. The two populations - as represented by twin MSs and SGBs - are traced up to more than 2.85 tidal radii from the cluster centre, incidentally contradicting the finding of Zoccali et al. (2009) of a strong radial gradient for the SGB. Carballo-Bello \& Martinez-Delgado (2010) detected with the ESO-WFI a clearly traceable MS of extra-tidal stars around NGC 1851 and NGC 1904. It is interesting to note that both studies do not find evidence of any collimated structure and both conclude that there is no evidence of tidal tails: NGC 1851 is simply embedded in a smooth, giant cloud of tidal debris.

According to Carballo-Bello \& Martinez-Delgado, it is tempting to associate this overdensity with the elusive Canis Major dwarf system: this conclusion would be corroborated by the observation that the old and metal-poor population of the debris of this system cover a sky-projected distance of about $3.3 \mathrm{kpc}$, similar to the distance between NGC 1851 and NGC 1904. Both clusters are considered (with NGC 2298 and NGC 2808) to be part of the Canis Major system (Martin et al. 2004). On the other hand, Olszewski et al. (2009) conclude that from existing dynamical models it is not possible to discriminate between isolated cluster evaporation and the destruction of a dwarf galaxy, when determining the origin of the observed giant halo around NGC 1851.

Is this cluster an exception? NGC 1851 shares several characteristics with the more metal-poor cluster M 22. Marino et al. $(2009,2011)$ found two sub-populations in this cluster differing in terms of both $\mathrm{Fe}$ and $\mathrm{Ba}$, with the abundances of $s$-process elements correlated with the iron abundance. The metallicity spread they found ( 0.10 dex rms scatter) does not differ much from the values found by either ourselves using high resolution spectra (0.07 dex) or Yong \& Grundahl (2008; 0.084 dex) for NGC 1851, and the metal-rich population is also Ca-rich with respect to the metal-poor one.

Finally, we note that the merger hypothesis was proposed by Lane et al. (2010a) to account for some kinematic features that could not be clearly explained for the GC 47 Tuc. For example, Lane et al. (2010b) note that a merger between two clusters of similar metallicity "would have increased the total luminosity of 47 Tuc without altering its $M / L_{V}$ significantly": this results in the cluster being an outlier in their $M / L_{V}$ vs. $M_{V}$ relation. However, a two component model containing populations with two distinct sets of kinematics is more applicable to 47 Tuc, whereas no significant kinematical difference can be established between the two populations in NGC 1851, apart from the observed radial segregation of stars on the RGB.

It is thus just possible that several channels are viable for the formation of different "flavours" of GC. Beside the large, massive GCs spiralling in the nucleus of their own galaxy, finding only a nuclear diffuse component, as in the case of M 54 and perhaps $\omega$ Cen (e.g. Carretta et al. 2010b), another channel is possibly feasible: the merger of two GCs in the same parent galaxy before the disruption within a much larger galaxy.

However, more observations are needed to settle the case of NGC 1851. In the future, we plan to analyse $\mathrm{CN}$ and $\mathrm{CH}$ low resolution spectra of SGB stars in each of the two SGBs found in NGC 1851 to investigate the role of the $\mathrm{C}$ and $\mathrm{N}$ abundances.
We also intend to test our hypothesis of a cluster merger by exploring the $\mathrm{Na}-\mathrm{O}$ anticorrelation in both the BHB and RHB of this intriguing cluster.

Acknowledgements. We thank Jae-Woo Lee for sending us his data on NGC 1851 in advance of publication, Antonio Sollima for useful discussions on the tidal remains around this cluster, Santino Cassisi for estimates of the maximum temperature along the HB for M 54 and $\omega$ Cen, and Yazan Momany for sending us his unpublished photometry of NGC 1851. We also thank the referee, whose suggestions helped us to improve the paper. This publication makes use of data products from the Two Micron All Sky Survey, which is a joint project of the University of Massachusetts and the Infrared Processing and Analysis Center/California Institute of Technology, funded by the National Aeronautics and Space Administration and the National Science Foundation. This research has been funded by PRIN MIUR 20075TP5K9, and by PRIN INAF "Formation and Early Evolution of Massive Star Clusters". This research has made use of the SIMBAD database, operated at CDS, Strasbourg, France and of NASA's Astrophysical Data System.

\section{Appendix A: Error estimates}

We refer the reader to the analogous appendices in Carretta et al. $(2009 a, b)$ for a detailed discussion of the procedure adopted for error estimates. In the following, we only provide the main tables of sensitivities of abundance ratios to the adopted errors in the atmospheric parameters and $E W \mathrm{~s}$ and the final estimates of internal and systematic errors for all species analysed using the UVES and GIRAFFE spectra of stars in NGC 1851 presented in this paper.

The sensitivities of the derived abundances to the adopted atmospheric parameters were obtained by repeating our abundance analysis and changing only one atmospheric parameter at each iteration for all stars in NGC 1851 and taking the average value of the slope change vs. abundance. This exercise was done separately for both UVES and GIRAFFE spectra.

When estimating the contribution to the internal errors of both the $E W \mathrm{~s}$ and $v_{\mathrm{t}}$, the values usually adopted (determined from the scatter in the abundances derived from individual lines) are overestimated, because regularities in the data are not taken into account. These regularities are for instance due to uncertainties in the $g f$-values or unrecognised blends with adjacent lines, a non-appropriate positioning of the continuum, etc. They manifest themselves in terms of uniform deviations of individual lines from average abundances for each star. We averaged over all stars the residuals of abundances derived from individual lines with respect to the average value for each star, and then estimated that some $36 \%$ of the total variance in the Fe abundances from individual lines is due to systematic offsets between different lines, which are then repeated from star-to-star. For about $30 \%$ of the lines, these offsets have trends with temperature that are significant at about the $2 \sigma$ level. However, we found that the additional fraction of variance that can be explained by these trends is very small, and can be neglected. We conclude that when considering star-to-star variations (internal errors, according to our denomination), the errors in $E W \mathrm{~s}$ and $v_{\mathrm{t}}$ should be multiplied by 0.8 .

The amount of variations in the atmospheric parameters is shown in the first line of the headers of Tables A.1 and A.2, whereas the resulting response to abundance changes of all elements (the sensitivities) are shown in Cols. 3-6 of these tables. 
Table A.1. Sensitivities of abundance ratios to both variations in the atmospheric parameters and errors in the equivalent widths, and errors in abundances for stars in NGC 1851 observed with UVES.

\begin{tabular}{|c|c|c|c|c|c|c|c|c|}
\hline Element & $\begin{array}{l}\text { Average } \\
\text { N. lines }\end{array}$ & $\begin{array}{l}T_{\text {eff }} \\
(\mathrm{K})\end{array}$ & $\begin{array}{l}\log g \\
(\operatorname{dex})\end{array}$ & $\begin{array}{c}{[\mathrm{A} / \mathrm{H}]} \\
(\mathrm{dex})\end{array}$ & $\begin{array}{c}v_{\mathrm{t}} \\
\mathrm{km} \mathrm{s}^{-1}\end{array}$ & $\begin{array}{l}E W s \\
(\text { dex })\end{array}$ & $\begin{array}{c}\text { Total } \\
\text { internal }\end{array}$ & $\begin{array}{c}\text { Total } \\
\text { systematic }\end{array}$ \\
\hline Variation & & 50 & 0.20 & 0.10 & 0.10 & & & \\
\hline Internal & & 4 & 0.04 & 0.06 & 0.04 & 0.070 & & \\
\hline Systematic & & 36 & 0.06 & 0.03 & 0.01 & & & \\
\hline$[\mathrm{Fe} / \mathrm{H}]_{\mathrm{I}}$ & 82 & +0.039 & +0.024 & +0.006 & -0.034 & +0.008 & 0.017 & 0.033 \\
\hline$[\mathrm{Fe} / \mathrm{H}]_{\mathrm{II}}$ & 9 & -0.063 & +0.107 & +0.036 & -0.015 & +0.023 & 0.039 & 0.064 \\
\hline$[\mathrm{O} / \mathrm{Fe}]_{\mathrm{I}}$ & 2 & -0.019 & +0.060 & +0.028 & +0.034 & +0.048 & 0.054 & 0.071 \\
\hline$[\mathrm{Na} / \mathrm{Fe}]_{\mathrm{I}}$ & 4 & +0.017 & -0.056 & -0.039 & +0.016 & +0.034 & 0.043 & 0.074 \\
\hline$[\mathrm{Mg} / \mathrm{Fe}]_{\mathrm{I}}$ & 3 & -0.007 & -0.017 & -0.008 & +0.019 & +0.039 & 0.040 & 0.011 \\
\hline$[\mathrm{Al} / \mathrm{Fe}] \mathrm{I}$ & 2 & +0.012 & -0.029 & -0.014 & +0.031 & +0.048 & 0.051 & 0.066 \\
\hline$[\mathrm{Si} / \mathrm{Fe}]_{\mathrm{I}}$ & 9 & -0.055 & +0.021 & +0.008 & +0.025 & +0.023 & 0.026 & 0.043 \\
\hline$[\mathrm{Ca} / \mathrm{Fe}]_{\mathrm{I}}$ & 17 & +0.037 & -0.037 & -0.020 & -0.022 & +0.016 & 0.023 & 0.030 \\
\hline$[\mathrm{Sc} / \mathrm{Fe}]_{\mathrm{II}}$ & 8 & +0.053 & -0.028 & -0.006 & -0.019 & +0.024 & 0.026 & 0.045 \\
\hline$[\mathrm{Ti} / \mathrm{Fe}]_{\mathbf{I}}$ & 9 & +0.058 & -0.030 & +0.008 & -0.003 & +0.023 & 0.024 & 0.047 \\
\hline$[\mathrm{Ti} / \mathrm{Fe}]_{\mathrm{II}}$ & 1 & +0.042 & -0.021 & -0.003 & +0.009 & +0.068 & 0.068 & 0.034 \\
\hline [V/Fe]I & 8 & +0.072 & -0.018 & -0.003 & +0.007 & +0.024 & 0.025 & 0.057 \\
\hline$[\mathrm{Cr} / \mathrm{Fe}]_{\mathrm{I}}$ & 3 & +0.029 & -0.025 & -0.017 & +0.018 & +0.039 & 0.041 & 0.026 \\
\hline$[\mathrm{Cr} / \mathrm{Fe}]_{\mathrm{II}}$ & 12 & +0.019 & -0.030 & -0.018 & +0.003 & +0.020 & 0.023 & 0.031 \\
\hline$[\mathrm{Mn} / \mathrm{Fe}]_{\mathrm{I}}$ & 3 & +0.021 & -0.015 & -0.009 & -0.001 & +0.039 & 0.040 & 0.025 \\
\hline$[\mathrm{Co} / \mathrm{Fe}] \mathrm{I}$ & 5 & -0.009 & +0.002 & +0.002 & +0.024 & +0.030 & 0.032 & 0.017 \\
\hline$[\mathrm{Ni} / \mathrm{Fe}]_{\mathrm{I}}$ & 29 & -0.018 & +0.017 & +0.007 & +0.015 & +0.013 & 0.015 & 0.017 \\
\hline$[\mathrm{Cu} / \mathrm{Fe}]_{\mathrm{I}}$ & 1 & +0.011 & -0.074 & +0.044 & -0.084 & +0.068 & 0.081 & 0.032 \\
\hline$[\mathrm{Y} / \mathrm{Fe}]_{\mathrm{II}}$ & 5 & +0.041 & -0.007 & +0.008 & -0.085 & +0.030 & 0.046 & 0.052 \\
\hline$[\mathrm{Zr} / \mathrm{Fe}] \mathrm{I}$ & 4 & +0.087 & -0.020 & -0.018 & +0.027 & +0.034 & 0.038 & 0.074 \\
\hline$[\mathrm{Zr} / \mathrm{Fe}]_{\mathrm{II}}$ & 1 & +0.092 & -0.099 & -0.036 & -0.087 & +0.068 & 0.082 & 0.079 \\
\hline$[\mathrm{Ba} / \mathrm{Fe}]_{\mathrm{II}}$ & 4 & +0.083 & -0.050 & +0.004 & -0.120 & +0.034 & 0.060 & 0.096 \\
\hline$[\mathrm{La} / \mathrm{Fe}]_{\mathrm{II}}$ & 2 & +0.110 & -0.180 & -0.036 & -0.035 & +0.048 & 0.066 & 0.106 \\
\hline$[\mathrm{Ce} / \mathrm{Fe}]_{\mathrm{II}}$ & 1 & +0.063 & -0.027 & -0.007 & -0.005 & +0.068 & 0.069 & 0.072 \\
\hline$[\mathrm{Nd} / \mathrm{Fe}]_{\mathrm{II}}$ & 2 & +0.073 & -0.043 & -0.011 & -0.105 & +0.048 & 0.065 & 0.069 \\
\hline$[\mathrm{Eu} / \mathrm{Fe}]_{\mathrm{II}}$ & 2 & +0.110 & -0.087 & +0.014 & +0.010 & +0.048 & 0.053 & 0.089 \\
\hline$[\mathrm{Dy} / \mathrm{Fe}]_{\mathrm{II}}$ & 1 & +0.078 & -0.026 & -0.002 & -0.005 & +0.068 & 0.069 & 0.066 \\
\hline
\end{tabular}

Table A.2. Sensitivities of abundance ratios to both variations in the atmospheric parameters and errors in the equivalent widths, and errors in abundances for stars in NGC 1851 observed with GIRAFFE.

\begin{tabular}{lcccccccc}
\hline \hline Element & $\begin{array}{c}\text { Average } \\
\text { N. lines }\end{array}$ & $\begin{array}{c}T_{\text {eff }} \\
(\mathrm{K})\end{array}$ & $\begin{array}{c}\log g \\
(\mathrm{dex})\end{array}$ & $\begin{array}{c}{[\mathrm{A} / \mathrm{H}]} \\
(\mathrm{dex})\end{array}$ & $\begin{array}{c}v_{\mathrm{t}} \\
\mathrm{km} \mathrm{s}^{-1}\end{array}$ & $\begin{array}{c}E W \mathrm{~s} \\
(\mathrm{dex})\end{array}$ & $\begin{array}{c}\text { Total } \\
\text { internal }\end{array}$ & $\begin{array}{c}\text { Total } \\
\text { systematic }\end{array}$ \\
\hline Variation & & 50 & 0.20 & 0.10 & 0.10 & & & \\
Internal & & 4 & 0.04 & 0.03 & 0.14 & 0.111 & & \\
Systematic & & 51 & 0.06 & 0.06 & 0.02 & & & \\
\hline$[\mathrm{Fe} / \mathrm{H}]_{\mathrm{I}}$ & 31 & +0.056 & -0.006 & -0.002 & -0.035 & +0.020 & 0.043 & 0.057 \\
{$[\mathrm{Fe} / \mathrm{H}]_{\mathrm{II}}$} & 3 & -0.032 & +0.091 & +0.024 & -0.012 & +0.064 & 0.057 & 0.043 \\
{$[\mathrm{O} / \mathrm{Fe}]_{\mathrm{I}}$} & 1 & -0.045 & +0.090 & +0.033 & +0.036 & +0.111 & 0.100 & 0.059 \\
{$[\mathrm{Na} / \mathrm{Fe}]_{\mathrm{I}}$} & 3 & -0.013 & -0.029 & -0.013 & +0.023 & +0.064 & 0.058 & 0.035 \\
{$[\mathrm{Mg} / \mathrm{Fe}]_{\mathrm{I}}$} & 2 & -0.022 & -0.003 & -0.004 & +0.021 & +0.078 & 0.067 & 0.023 \\
{$[\mathrm{Si} / \mathrm{Fe}]_{\mathrm{I}}$} & 7 & -0.053 & +0.034 & +0.009 & +0.029 & +0.042 & 0.048 & 0.055 \\
{$[\mathrm{Ca} / \mathrm{Fe}]_{\mathrm{I}}$} & 5 & +0.003 & -0.030 & -0.005 & -0.002 & +0.050 & 0.040 & 0.010 \\
{$[\mathrm{Sc} / \mathrm{Fe}]_{\mathrm{II}}$} & 5 & -0.058 & +0.087 & +0.029 & +0.013 & +0.050 & 0.047 & 0.065 \\
{$[\mathrm{Ti} / \mathrm{Fe}]_{\mathrm{I}}$} & 4 & +0.020 & -0.004 & -0.010 & +0.015 & +0.056 & 0.048 & 0.021 \\
{$[\mathrm{~V} / \mathrm{Fe}]_{\mathrm{I}}$} & 6 & +0.030 & -0.001 & -0.010 & +0.020 & +0.045 & 0.043 & 0.033 \\
{$[\mathrm{Cr} / \mathrm{Fe}]_{\mathrm{I}}$} & 4 & +0.003 & -0.002 & -0.008 & +0.026 & +0.056 & 0.053 & 0.012 \\
{$[\mathrm{Co} / \mathrm{Fe}]_{\mathrm{I}}$} & 1 & +0.004 & +0.019 & +0.005 & +0.025 & +0.111 & 0.093 & 0.014 \\
{$[\mathrm{Ni} / \mathrm{Fe}]_{\mathrm{I}}$} & 7 & -0.015 & +0.024 & +0.005 & +0.018 & +0.042 & 0.040 & 0.018 \\
{$[\mathrm{Zr} / \mathrm{Fe}]_{\mathrm{I}}$} & 2 & +0.066 & -0.013 & -0.014 & +0.031 & +0.078 & 0.072 & 0.086 \\
{$[\mathrm{Ba} / \mathrm{Fe}]_{\mathrm{II}}$} & 1 & -0.043 & +0.041 & +0.044 & -0.030 & +0.111 & 0.097 & 0.051 \\
\hline
\end{tabular}

\section{References}

Alonso, A., Arribas, S., \& Martinez-Roger, C. 1999, A\&AS, 140, 261 Alonso, A., Arribas, S., \& Martinez-Roger, C. 2001, A\&A, 376, 1039 Anderson, J. 1998, Ph.D.. Thesis, Univ. California, Berkeley Arnould, M., Goriely, S., \& Jorissen, A. 1999, A\&A, 347, 572 Bertelli, G., Girardi, L., Marigo, P., \& Nasi, E. 2008, A\&A, 484, 815 Biemont, E., Grevesse, N., Hannaford, P., \& Lowe, R. M. 1981, ApJ, 248, 867
Calamida, A., Bono, G., Stetson, P. B., et al. 2007, ApJ, 670, 400

Carballo-Bello, J. A., \& Martinez-Delgado, D. 2010, in Highlights of Spanish Astrophysics V (Berlin, Heidelberg: Springer-Verlag), ASSP Proc., 383

Bragaglia, A., Carretta, E., Gratton, R. G., et al. 2010, A\&A, 519, 60 Carretta, E. 2006, AJ, 131, 1766

Carretta, E., Bragaglia, A., Gratton, R. G., et al. 2006, A\&A, 450, 523

Carretta, E., Bragaglia, A., Gratton, R. G., Lucatello, S., \& Momany, Y. 2007a, A\&A, 464, 927 
Carretta, E., Bragaglia, A., Gratton, R. G., et al. 2007b, A\&A, 464, 939 Carretta, E., Bragaglia, A., Gratton, R. G., et al. 2007c, A\&A, 464, 967 Carretta, E., Bragaglia, A., Gratton, R. G., et al. 2009a, A\&A, 505, 117

Carretta, E., Bragaglia, A., Gratton, R. G., \& Lucatello, S. 2009b, A\&A, 505, 139

Carretta, E., Bragaglia, A., Gratton, R. G., D’Orazi, V., \& Lucatello, S. 2009c, A\&A, 508, 695

Carretta, E., Bragaglia, A., Gratton, R. G., et al. 2010a, A\&A, 516, A55

Carretta, E., Bragaglia, A., Gratton, R. G., et al. 2010b, ApJ, 714, L7

Carretta, E., Gratton, R. G., Lucatello, S., et al. 2010c, ApJ, 722, L1

Carretta, E., Bragaglia, A., Gratton, R., et al. 2010d, ApJ, 712, L21

Carretta, E., Bragaglia, A., Gratton, R. G., et al. 2010e, A\&A, 520, A95

Carretta, E., Bragaglia, A., Gratton, R. G., D’Orazi, V., \& Lucatello, S. 2011, A\&A, submitted

Cassisi, S., Salaris, M., Pietrinferni, A., et al. 2008, ApJ, 672, L11

Catelan, M. 1997, ApJ, 478, L99

Cohen, J. G. 1978, ApJ, 223, 487

Cohen, J. G. 2004, AJ, 127, 1545

Conroy, C. 2011 [arXiv: 1101.2208]

D’Antona, F., Bellazzini, M., Caloi, V., et al. 2005, ApJ, 631, 868

D'Antona, F., D'Ercole, A., Marino, A. F., et al. 2011, ApJ, 736, 5

Decressin, T., Meynet, G., Charbonnel, C., Prantzos, N., \& Ekstrom, S. 2007, A\&A, 464, 1029

Denisenkov, P. A., \& Denisenkova, S. N. 1989, A. Tsir., 1538, 11

D’Ercole, A., D'Antona, F., Ventura, P., Vesperini, E., \& McMillan, S. L. W. 2010, MNRAS, 407, 854

D’Orazi, V., Gratton, R. G., Pancino, E., et al. 2011, A\&A, submitted

Gratton, R. G., Carretta, E., Eriksson, K., \& Gustafsson, B. 1999, A\&A, 350, 955

Gratton, R. G., Sneden, C., Carretta, E., \& Bragaglia, A. 2000, A\&A, 354, 169

Gratton, R. G., Bonifacio, P., Bragaglia, A., et al. 2001, A\&A, 369, 87

Gratton, R. G., Carretta, E., Claudi, R., Lucatello, S., \& Barbieri, M. 2003, A\&A, 404, 187

Gratton, R. G., Sneden, C., \& Carretta, E. 2004, ARA\&A, 42, 385

Gratton, R. G., Carretta, E., Bragaglia, A., Lucatello, S., \& D’Orazi, V. 2010a, Msngr., 142, 28

Gratton, R. G., Carretta, E., Bragaglia, A., Lucatello, S., \& D’Orazi, V. 2010b, A\&A, 517, A81

Han, S.-I., Lee, Y.-W., Joo, S.-J., et al. 2009, ApJ, 707, L190

Harris, W. E. 1996, AJ, 112, 1487

Ivans, I. I., Sneden, C., Kraft, R. P., et al. 1999, AJ, 118, 1273

Ivans, I. I., Kraft, R. P., Sneden, C., et al. 2001, AJ, 122, 1438

Johnson, C. I., \& Pilachowski, C. A. 2010, ApJ, 722, 1373

Käppeler, F. 1999, PPNP, 43, 419

Kraft, R. P. 1994, PASP, 106, 553
Kurucz, R. L. 1993, CD-ROM 13, Smithsonian Astrophysical Langer, G. E., Hoffman, R., \& Sneden, C. 1993, PASP, 105, 301 Lane, R. R., Brewer, B. J., Kiss, L. L., et al. 2010a, ApJ, 711, L122

Lane, R. R., Kiss, L. L., Lewis, G. F., et al. 2010b, MNRAS, 406, 2732 Lardo, C., Bellazzini, M., Pancino, E., et al. 2011, A\&A, 525, 114

Lee, J.-W., Lee, J., Kang, Y.-W., et al. 2009a, ApJ, 695, L78

Lee, J.-W., Kang, Y.-W., Lee, J., \& Lee, Y.-W. 2009b, Nature, 462, 480

MacQueen, J. B. 1967, Mathematical Statistics and Probability (University of California Press), 281

Magain, P. 1984, A\&A, 134, 189

Makino, J., Akiyama, K., \& Sugimoto, D. 1991, Ap\&SS, 185, 63

Marino, A. F., Villanova, S., Piotto, G., et al. 2008, A\&A, 490, 625

Marino, A. F., Milone, A. P., Piotto, G., et al. 2009, A\&A, 505, 1099

Marino, A.F., Sneden, C., Kraft, R.P. et al. 2011, A\&A, 532, A8

Martin, N. F., Ibata, R. A., Bellazzini, M., et al. 2004, MNRAS, 348, 12

Milone, A. P., Bedin, L. R., Piotto, G., et al. 2008, ApJ, 673, 241

Milone, A., Stetson, P. B., Piotto, G., et al. 2009, A\&A, 503, 755

Olszewski, E. W., Saha, A., Knezek, P., et al. 2009, AJ, 128, 1570

Peterson, R. C. 1980, ApJ, 237, L87

Prantzos, N., \& Charbonnel, C. 2006, A\&A, 458, 135

$\mathrm{R}$ Development Core Team 2011, R: A language and environment for statistical computing, Vienna, Asutria, ISBN 3-900051-07-0

Raiteri, C. M., Busso, M., Gallino, R., \& Picchio, G. 1991, ApJ, 371, 665

Recio-Blanco, A., Aparicio, A., Piotto, G., De Angeli, F., \& Djorgovski, S. G. 2006, A\&A, 452, 875

Salaris, M., Weiss, A., Ferguson, J. W., \& Fusilier, D. J. 2006, ApJ, 645, 1131

Sbordone, L., Bonifacio, P., Buonanno, R., et al. 2007, A\&A, 465, 815

Simmerer, J., Sneden, C., Cowan, J. J., et al. 2004, ApJ, 617, 1091

Skrutskie, M. F., Cutri, R. M., Stiening, R., et al. 2006, AJ, 131, 1163

Sneden, C., Gratton, R. G., \& Croker, D. A. 1991, A\&A, 246, 354

Sneden, C., McWilliam, A., Preston, G. W., et al. 1996, ApJ, 467, 819

Sneden, C., Cowan, J. J., Lawler, J. E., et al. 2003, ApJ, 591, 936

Steinhaus, H. 1956, Bull. Acad. Polon. Sci., 4, 801

van den Bergh, S. 1996, ApJ, 471, L31

Travaglio, C., Gallino, R., Arnone, E., et al. 2004, ApJ, 601, 864

Ventura, P., D’Antona, F., Mazzitelli, I., \& Gratton, R. 2001, ApJ, 550, L65

Villanova, S., Geisler, D., \& Piotto, G. 2010, ApJ, 722, 118 (V10)

Walker, A. 1992, PASP, 104, 1063

Yong, D., \& Grundahl, F. 2008, ApJ, 672, L29

Yong, D., Grundahl, F., Nissen, P. E., Jensen, H. R., \& Lambert, D. L. 2005 A\&A, 438, 875

Yong, D., Grundahl, F., Johnson, J. A., \& Asplund, M. 2008. ApJ, 684, 1159

Yong, D., Grundahl, F., D’Antona, F., et al. 2009, ApJ, 659, L62

Zoccali, M., Pancino, E., Catelan, M., et al. 2009, ApJ, 697, L22 